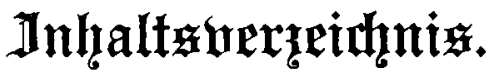

\section{Griter Band.}

Einleitung.

I. Gejđidute des Gejebes. . . . . . . . . . . . . . . . . . . . . . . . . . 23

II. Siteratur . . . . . . . . . . . . . . . . . . . . . . . . . . . 28

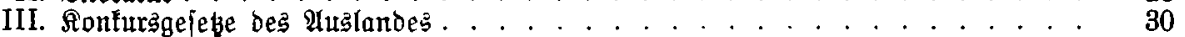

IV. ßaragraphenvergleiđung . . . . . . . . . . . . . . . . . . . . . . . . . 35

I.

包te

\author{
Erites $\mathfrak{B} \mathfrak{u}$ ). \\ finkuxurent. \\ Friter Titel. \\ arlgemeine Beptimmungen.
}

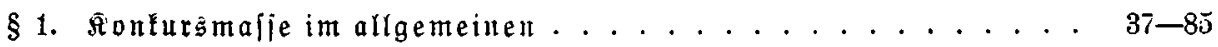

I. Sollmalie und જitmaife [भimm. 1-3].

II. Grenzen des Bermögens [ $\mathfrak{A}$ m. 4-17].

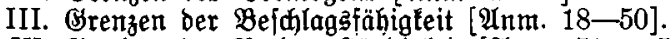

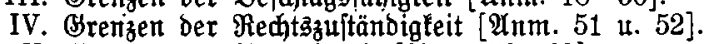

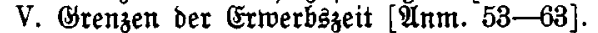

VI. Gejamt und Sonderfonfurs [भInm. 64-70].

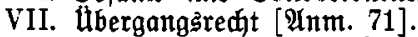

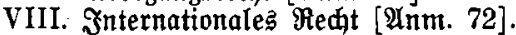

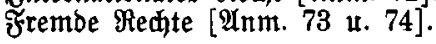

§ 2. Sonfursmafle einez Ehegatter. . . . . . . . . . . . . . . 86-101

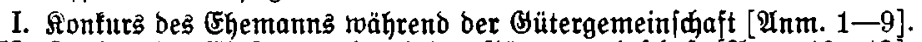

II. Ronkurs ber (Ehefrau währeno ber (Sütergemeiniałałt [Anm. 10-13].

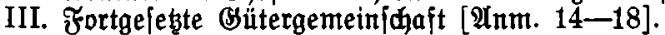

IV. Gonturs nađ Beendigung Der ehelidien ober Der fortgeießten Büter.

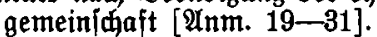

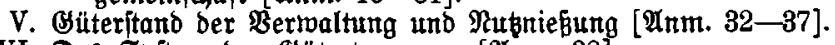

VI. Das Syitem ber Süütertrennung [भinm. 38].

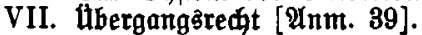

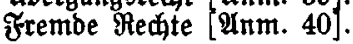


§3. Sonkurzgläubiger . . . . . . . . . . . . . . . . . 101-129

I. Begriff der Ronkuts̉gläubiger [QInm. 1-33].

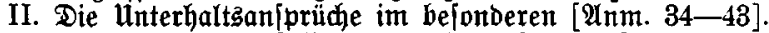

III. Beriprecten ber \&eiftung an einen Dritten [Änm. 44-46].

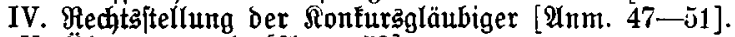

V. übergangšrecht [भ̈nm. 52].

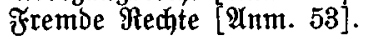

§4. $\mathfrak{A b}$ fonderungaredit . . . . . . . . . . . . . . 130-136

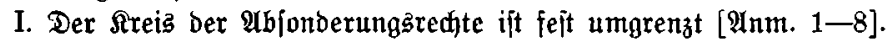

II. Albgejonderte Befriebigung erfolgt unabhängig vom sionfurs: verfahren [थ̈m. 9-11].

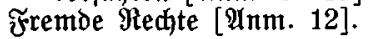

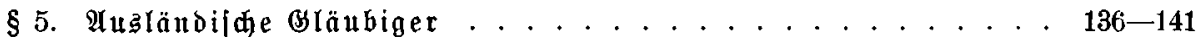

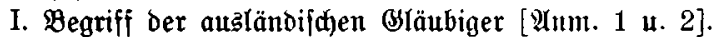

II. Die Regel [ $[\mathfrak{A n m} .3$ u. 4].

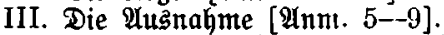

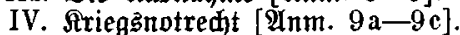

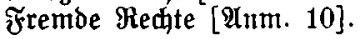

§6. Sonfurspertoalter . . . . . . . . . . . . . . . . 141-177

A. Ređttaftellung bes Rionfurs̄nerwalters.

I. Die Streitfrage [भinm. 1-4].

II. Die Entideisung [Mnm. 5-17].

B. Der Sinfuräberivalter alß gejeblidjer Bertreter.

I. Folgen Der Bertreterftellung Des Berwalters [Ânm. 18-22].

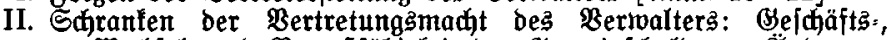

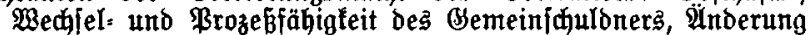
Der Ber[on des Berwalters, Freinillige Beridhtsbarteit [भ̊m. 23-37].

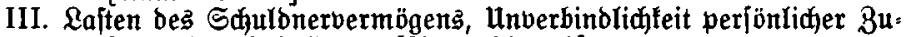
jagen bes Sduulbners [थีm. 38-40].

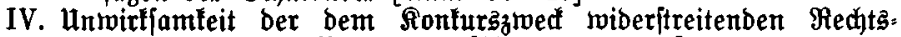
hanblungen bes $\mathfrak{B e r m a l t e r s ~ [ \mathfrak { A n m } . 4 1}$ u. 42].

V. Frreigabe von Maflegegenftänben [ $\mathfrak{A m} .43$ u. 44].

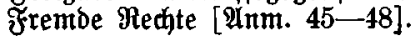

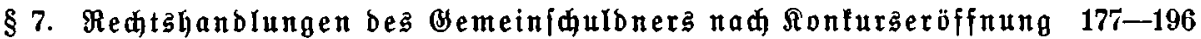

A. Die Regel [ $\mathfrak{A n m} .1-20]$.

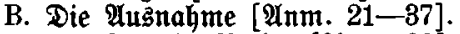

Frembe Redte [थ̈nm. 38].

§ 8. Seiftungen an ben Bemeinfđulbner . . . . . . . . . . 196-204

I. Die $\Re$ egel $[\mathfrak{A n m}$. 1-11].

II. Die $\mathfrak{A} u \mathfrak{m a h m e}[\mathfrak{A} \mathfrak{n m} .12-23]$.

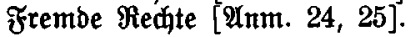

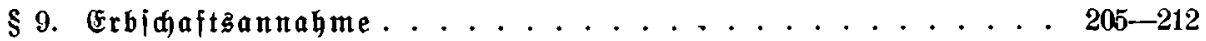

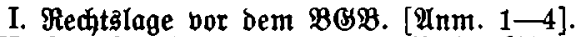

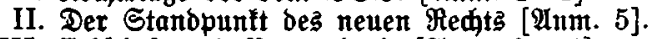

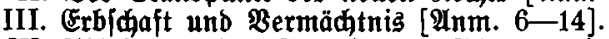

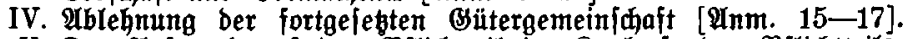

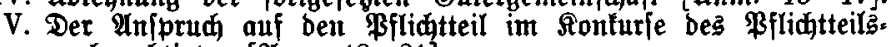
beredtigten [ânm. 18-21].

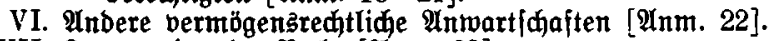

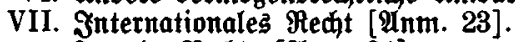

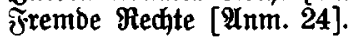

$\S 10$. Brozézunterbrequng . . . . . . . . . . . . . 212-234

A. Die Brozeßunterbređung im allgemeinen.

I. Brunbgebante [थ̈m. 1].

II. Die Borausfęungen Der Unterbrechung [थnm. 2-6].

III. Die Folgen ber unterbredung [थ̈nm. 7 u. 8].

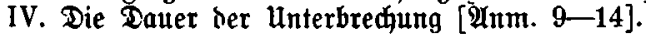


B. Aftioprozeile.

Eeite

I. Begriff Des Afttioprozelfes [Ânm. 15-24].

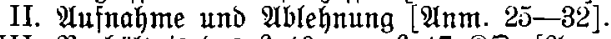

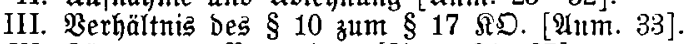

IV. 3̈̈gern Des Berwalters [\{̂nm. 34-37].

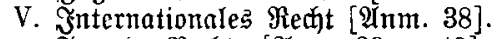
Frembe Redite [9nm. 39 u. 40].

\$11. Fajioprozelje.

$234-242$

I. Antuendungagebiet bes § 11 [Annt. 1-i].

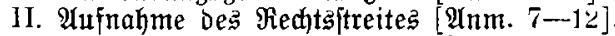

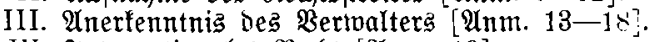

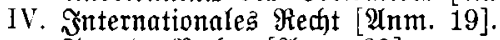
đrembe Rehte [Âtm. 20].

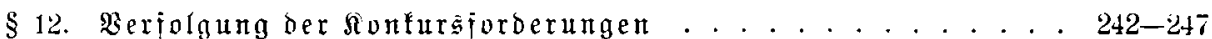

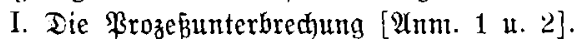

II. Die Bebeutung Des $\$ 12[91 \mathrm{~nm} .3-8]$. Frembe Ređte [:rm. 9].

$\S 13$. Beräujerungşerbot . . . . . . . . . . . . . . . 247-253

I. Megelfall [Änm. 1-7].

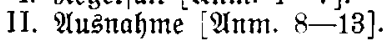

§ 14. Berbot ber Eillzeloollitredung . . . . . . . . . . . . 2ü3-268

A. 3ivangävollijtređ̛ng und $\mathfrak{A r r e j t . ~}$

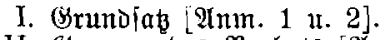

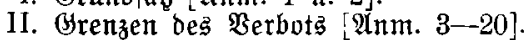

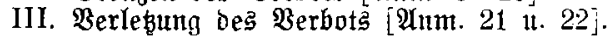

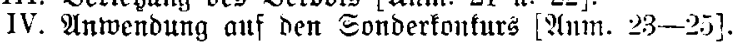

B. Die 3mangsvormerfung.

I. AIIgemeines [Anm. 26-29].

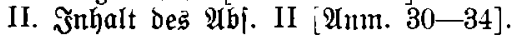

C. Internationales $\mathfrak{R e d}$ t $[\mathfrak{A n m}$. 35]. Fremoe Rechte [भinm. 36-39].

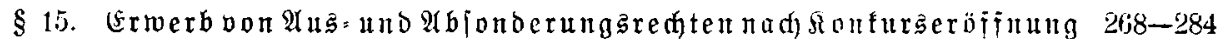

A. Die Regel.

I. Grenzen nad altem uno neuem Recht [\{̊m. 1-9].

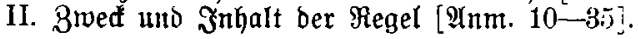

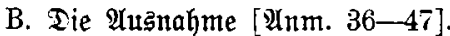

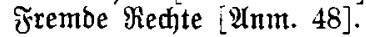

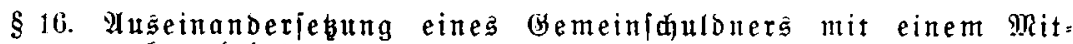

beredtigten . . . . . . . . . . . . . . 285-291

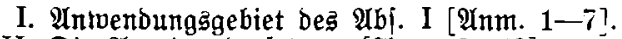

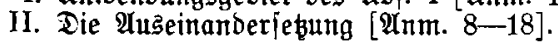

3reiter Titel.

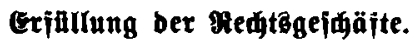

§ 17. Begenleitige $\mathfrak{B e r t r a ̈ g e ~ . ~ . ~ . ~ . ~ . ~ . ~ . ~ . ~ . ~ . ~ . ~ . ~ . ~ . ~ . ~ . ~ . ~ 2 9 1 - 3 2 9 ~}$

I. Anwentungşgebiet beș $\$ 17$ [अ̈nnt. 1-29].

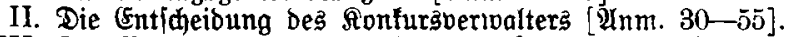

III. Der Berlagşuertrag im bejonberen [थ̈nn. $56-63]$.

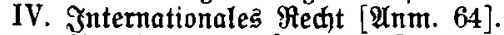
Frembe Redite [Ânm. 65].

$\S 18$. Firgeldăfte . . . . . . . . . . . . . . . . . 329-335

I. Ratio legis [थ̈nm. 1].

II. Borausfęungen bes $\$ 18$ [थाm. 2-14].

III. Jnhalt beş $\$ 18[\mathfrak{A}$ mm. 15-26]. Frembe Redite [भ̊m. 27]. 
§ 19. Bemeinjuldoner als Mieter (Mietgegenitand überlaflen) . . 336-347

I. Allgemeines zu Delt $\S \S 19-21$ [भum. 1 u. 2].

II. Borausjekzungen Deș $\$ 19$ [थ̛nm. 3-7].

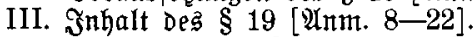
Frembe Rechte [भ̆nm. 23-25].

§ 20. Gemeinfduldor als Mieter (Mietgegenitand nod nidt über:

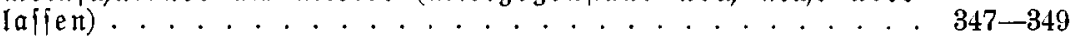

I. Die Borausjekungen beş $\$ 20$ [Ünm. 1].

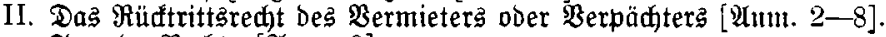

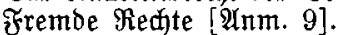

§ 21. Bemeinf

I. Borausjebungen bez $\S 21$ [भmm. 1].

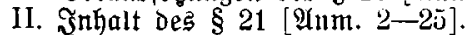

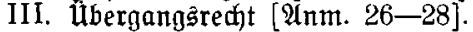
Frtembe Redite [Ânt. 29 น. 30].

§ 22. Tienftoerhältuijie . . . . . . . . . . . . . . . . . . . . . . . . 360-360

I. Borausfebungen bes § 22 [Arm. 1-10].

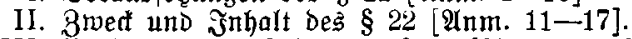

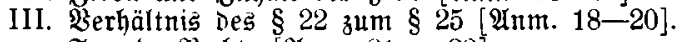
Frembe Redite $[9 \pi m .21$ u. 22$]$.

§ 23. (Gefdäftsbejorgung für den (semeiniduloner . . . . . . . . 309-384

A. Stonfurs bes Gejhäftsherrit.

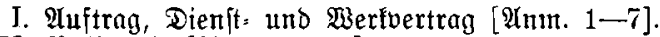

II. Bollmadyt [Ämm. 8-12].

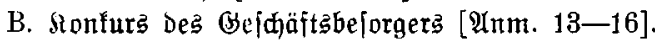

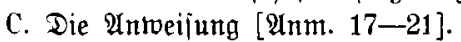

Fremtoe Redfte [भinm. 22].

$\S 24$. Bormertung

I. Die $\mathfrak{B}$ ormerfung im allgemeinen [ăm. 1-6].

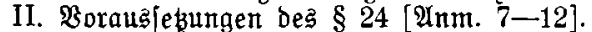

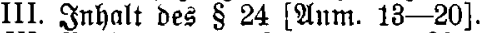

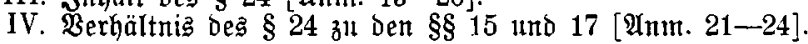
Frembe Redte $[\mathfrak{A m} .25]$.

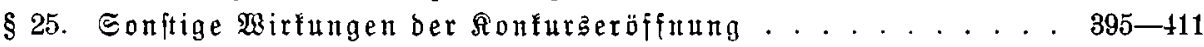

A. Bedeutung Des $\S 25$ [भInt. 1-5].

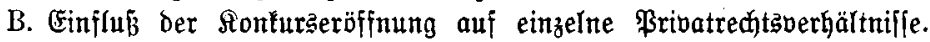

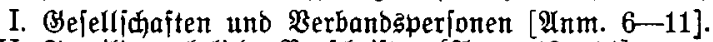

II. Familienredtlidue Boridriften [QInm. 12-14].

III. Unterbređung ber Berjährung [भ̆mm. 15-28].

IV. Wirfungen Der Gröffinung des Rahlaṕlonturtes insbejondere [भimm. 29-32].

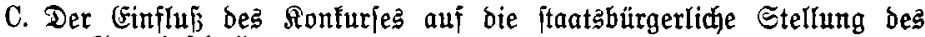
Bemeinifuuldnerş.

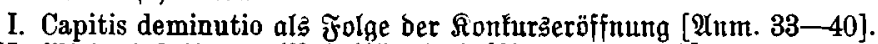

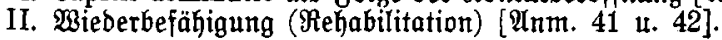

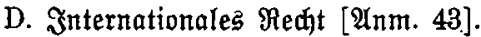
Frembe Redte [ $\mathfrak{A}$ mm. 44-47].

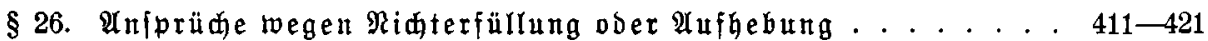

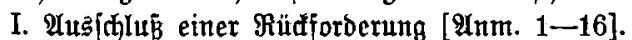

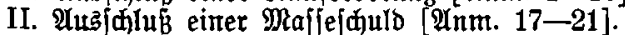

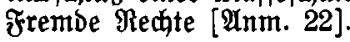

§ 27. Sefđäftşbeforger als Maffe= ober Ronturşgläubiger . . . . . 421-424

I. Der Gefdüftşbejorger als Maflegläubiger [

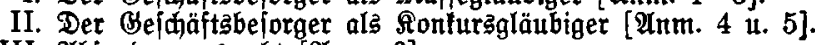

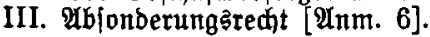


§28. Befellidafter alz Miale= ober Ronfurägläubiger . . . . . . 424-426

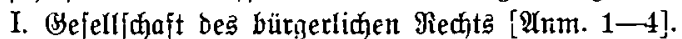

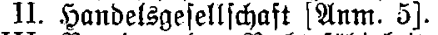

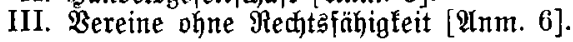
Frembe Rechte [भ̆m. 7].

\section{Dritter Titel.}

\section{צnfed̆tung.}

§ 29. Anjed)tung.

$426-480$

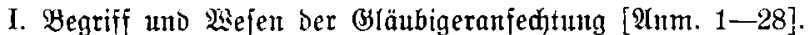

II. Der Anfechtungstatbeftand im allgemeinen [Ümt. 29-55].

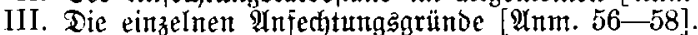

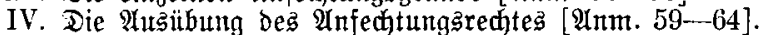

V. Beitlidge und răumliḑe Sctranten ber ßläubigeranfect)tung [?⿻𨈑口. $65-72]$.

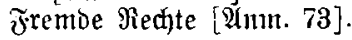

\$ 30. Die befondere Ronturzanfectung . . . . . . . . . . . . 487--531

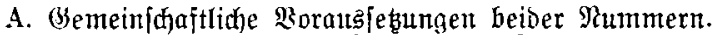

I. Bahlungseinitellung [9anm. 1-15].

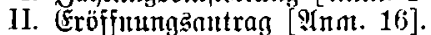

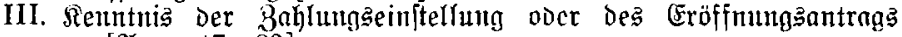
[थ̊tm. 17-23].

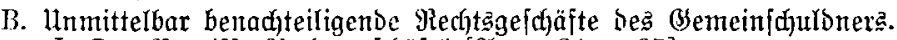

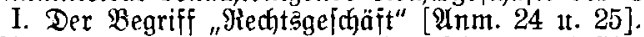

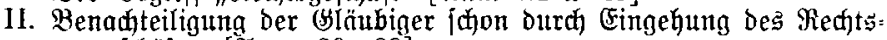

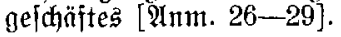

c. Dectung einer Sonfursforderung.

I. Bemeinjame Brundiäte für beide Dechungsarten [थrm. 30-43].

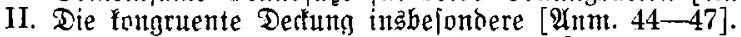

III. Die intongruente Dedung itsbejondere [9: Frembe Recte [थนm. 65].

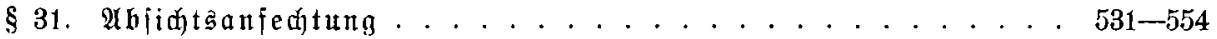

I. Megelfall (Mr. 1) [Minm. 1-19].

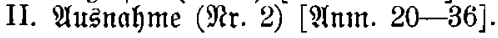

Frembe Rechte [थrm. 37].

$\S 32$. ङุenfungsanfechtung . . . . . . . . . . . . . 554-574

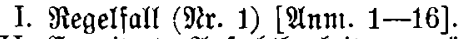

II. Erweiterte $\mathscr{M}$ nfedtbarfeit gegenüber bem Ghegatten (Mr. 2) [थ̈m. 17-22].

III. Berfpred)en Der Leijtung an einen Dritten [A̛m. 23-29]. Frembe Medite [Anm. 30].

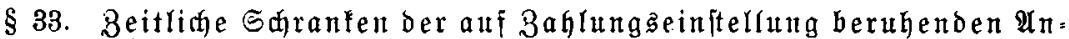

fedtbarket. . . . . . . . . . . 575-577

I. Boraus[etungen bes $\S 33$ [ânm. 1 u. 2].

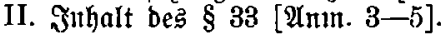

Frembe Rechte [थ̈nm. 6].

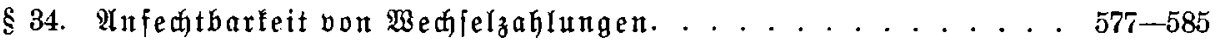

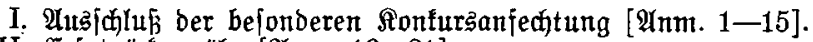

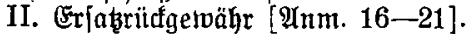

III. Bahlung auf eiten Shyed [भim. 22-24]. Frentbe Redite [Anm. 25].

$\S 35$. Bolltredtbare Titel und Bollfitredungen . . . . . . . . . . . 585-588

I. Die Brundiäte bes $\$ 35$ [Mrnm. 1-4].

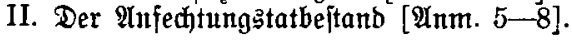
Frembe Medte [थmm. 9]. 
Eeite

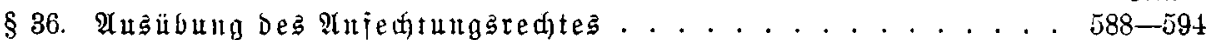

I. Ermäntigung deș Berwalters [ $\mathfrak{A} \mathfrak{n m}$. 1-3].

II. Zroangavertretung Des $\mathfrak{M a}$ afeträaers [2̈nm. 4-9].

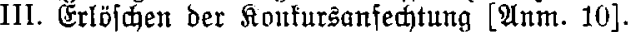

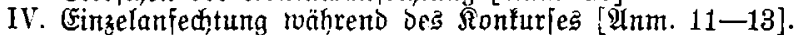

V. Ċinfluf Der fionfuršeröffruntg und Sonfursbeendigung auf bie

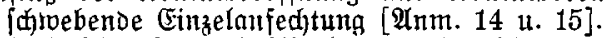

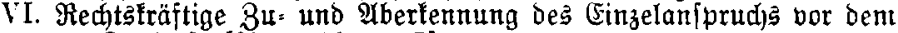

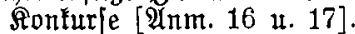
Frembe Rectite [भIm. 18].

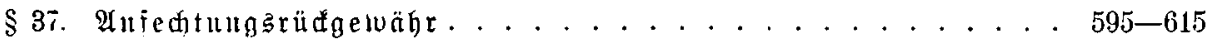

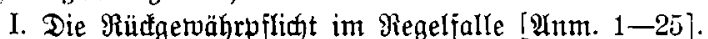

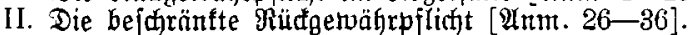

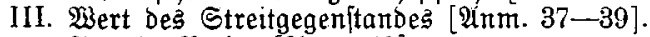
Frembe Redite [A̛m. 40$]$ ].

$\$ 38$. Erftattung eiter Begenleijtung. . . . . . . . . . . . . 616-620

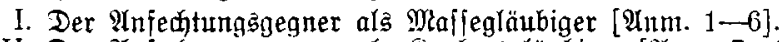

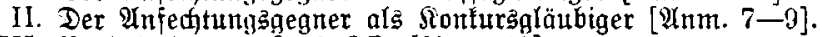

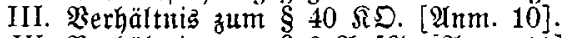

IV. Berhăltnis zum \& 8 भris. [MInm. 11]. Frembe Redte [Mnm. 12].

§ 39. Wiederaufleben eines Gegenaniprudis. . . . . . . . . . . . . . 620-624

I. Bedeutung Dcs $\$ 39$ [Änt. 1].

II. Boraus[ekungen bes $\$ 39$ [भinn. 2-7].

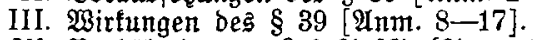

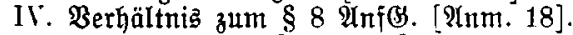

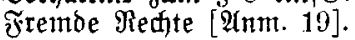

\$ 40. Mufeditbarfeit gegenüher geditsnadfolgern . . . . . . . . . 624-640

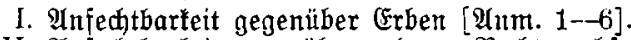

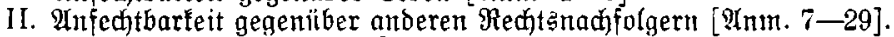
Frembe Ned)te [în. 30].

§ 41. Beitlide ธquranten ber Anfeditung . . . . . . . . . . . 640-647

I. Die redtliche Ratur Der 2tufedtungsfriften [9nm. 1 u. 2].

II. Der Friftenlauf [Anm. 3-6].

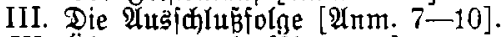

IV. $\mathfrak{a}$ Gergangaredgt $[\mathfrak{A}$ m. 11]. Frembe Rechte [भinm. 12]

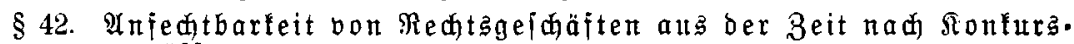
eröffutıg . . . . . . . . . . . . . . .

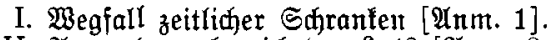

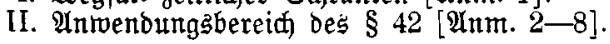

\section{Bierter Titel.}

\section{Anßojonberung.}

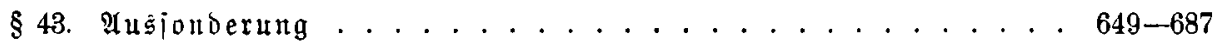

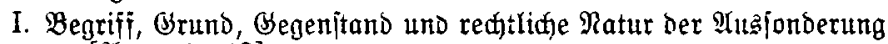
[\{̈ 1 m. 1-13].

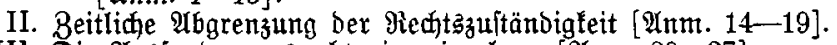

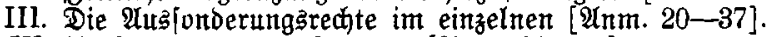

IV. Aus [onberuttg von Treugut [92m. 38-47].

V. Be[onderheiten bet Berträgen für frembc Rechnung [भrmm. 48-55].

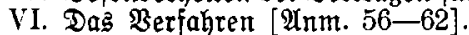

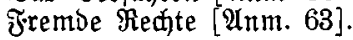

§44. Berfolgungšedt. . . . . . . . . . . . . . 687-701

I. Die Bedeutung bes $\S 44$ [थnm. 1-4].

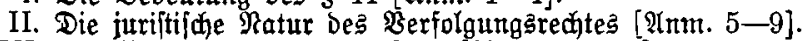

III. Die Borausfetungen bes $\$ 44$ [Mrm. 10-22]. 


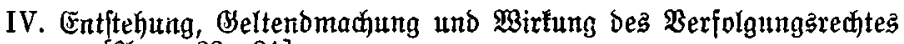
$[\mathfrak{A} \mathfrak{n m} .23-31]$.

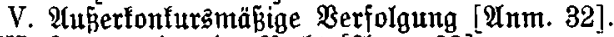

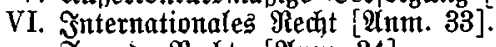

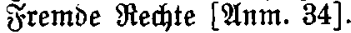

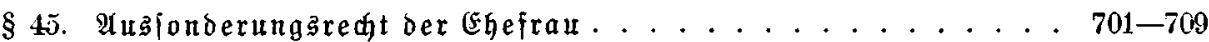

I. Bwed uno Bebeutung bes $\$ 45$ [भnm. 1-4].

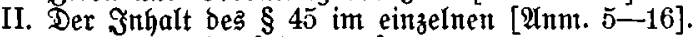
Frembe Otedte [\{̈nm. 17].

§46. Erịąałsjonderung.

I. Bebeutung des $\$ 46$ [:Hin. 1-5].

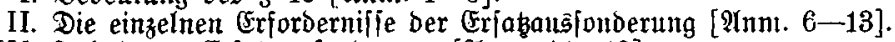

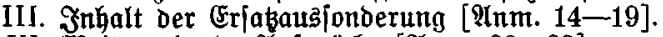

IV. Beitergehende $\mathscr{A}$ ipriidse $[\mathfrak{A n m} .20-22]$.

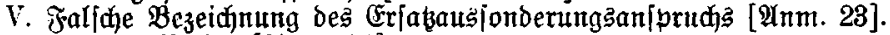
Frembe Redite [भnm. 24].

\section{路üfter Titel.}

\section{abjonberung.}

§47. 2(bjonderung . . . . . . . . . . . . . . . . 721-736

I. Die $\mathfrak{A b}$ onderung in allgemeinen [Anm. 1-15].

II. ÂGgejonberte $\mathfrak{B e f r i e b i g u n g ~ a u s ~ u n b e r v e g l i d e m ~ B e r m o ̈ g e n ~}$ [भInm. 16-28].

III. Internationales $\mathfrak{R e d t}[\mathfrak{A}$ nm. 29].

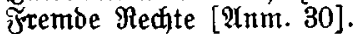

$\S$ 48. Uhgejouberte Befriebigurg aus betweglidem Bermb̈gen (Anred)=

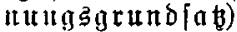

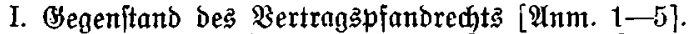

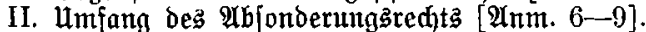

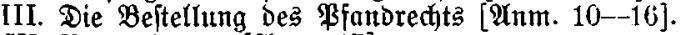

IV. Rattgorontung [2Inm. 17].

V. Znternationales Recht. Frembe Redte [Äm. 18]

\$4. Seleblitie Uyjonderungredite.

I. Das $\mathfrak{A b g a b e n a b j o n d e r u n g s ̧ r e d ~}[$ [nm. 1-3].

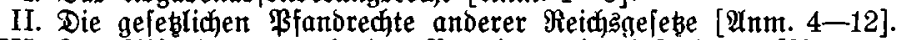

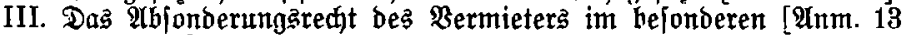
bia 31].

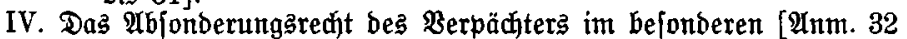
bis3 35].

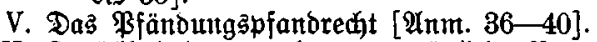

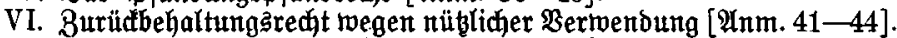

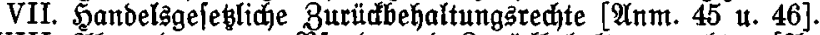

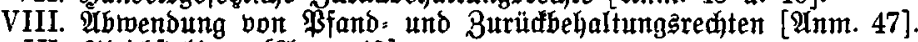

IX. Bileidiftellung [29mm. 48].

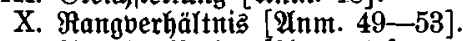
Frembe Redte [Artm. 54].

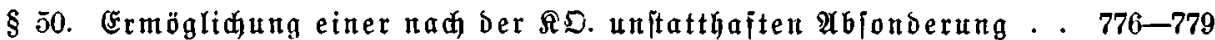

I. Ratio legis [ $\mathfrak{A} \mathfrak{n m} .1$ ].

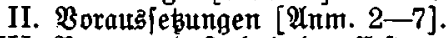

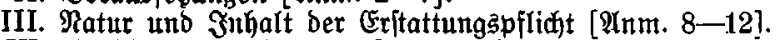

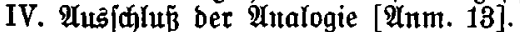

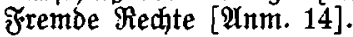

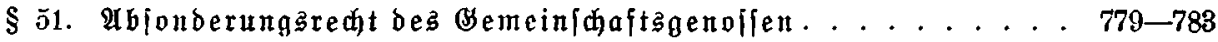

I. Bebeutung und Beltungăbereich des $§ 51$ [भinm. 1-3].

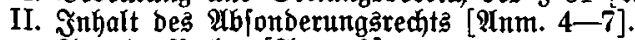
Fremto Redite [भinm. 8].

§52. Ábjonderung aủ $\mathfrak{L}$ ehen, Stammgütern, Fibeifommiffen . . . 783-784 
$\S$ i3. $\mathfrak{A} u \mathfrak{j}$ redinung . . . . . . . . . . . . . . . . . . . . . . . . $784-802$

1. Die Brnutoläbe ber $\mathfrak{A} u f r e c h n u n g ~[\mathscr{A n m} .1-23]$.

II. Geltenomad)ung ber $\mathfrak{A}$ ufred)nung im Sonkurs [Âm. 24-26].

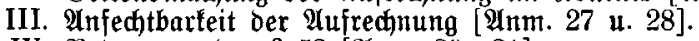

IV. Bebeutung bez $\S 53$ [भItm. $29-31]$.

V. Grenzen ber fonturstedtlid)en Sonderboridriften [\{1⿴m. 32-34].

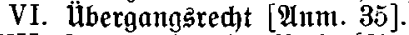

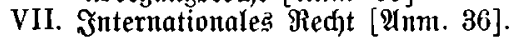

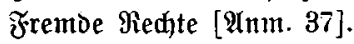

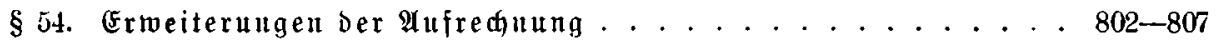

I. 3roet und Grenzen iesz $\S 54$ [\$(nm. 1].

II. Betagte Frorberungen [:nm. 2-4].

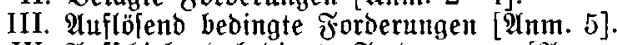

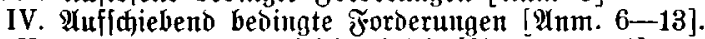

V. Erfordernis ber Bleidgartigleit [Anm. 14-16].

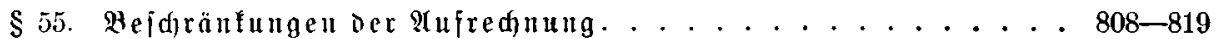

I. Bedeutultg Des $\$ 55$ im allgeneinen [2utm. 1-3].

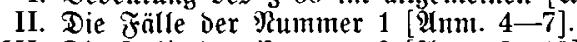

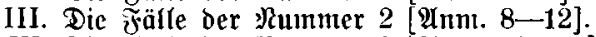

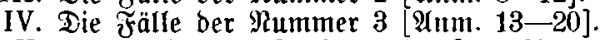

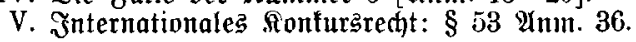
Frembe Rechte: ebention $211 m .37$.

§56. Errabpflidt twegen Ermöglidung einer nad ber $\mathfrak{R}$ D. unftatthajten

$\mathfrak{A} u$ freduung. . . . . . . . . . . . .

\section{Siebenter Titel.}

\section{Mafiegläubiger.}

$\S \overline{5}$. Borluegbefriebigung ber Miallegläubiger

$820-829$

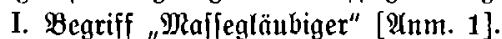

II. Sdulloner ber Mafiegläubiger [भnm. 2-9].

III. Berfolgung ber Maffeaniprüche [भitm. $10-12$ ]

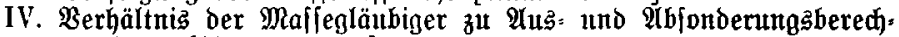
tigten $[\mathfrak{H}$ mm. 13-15].

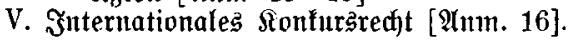
Frembe Mechte [भnm. 17].

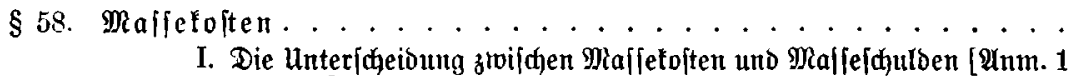
u. 2].

II. Die einzelner Majfelojten [Änm. 3-8].

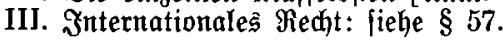

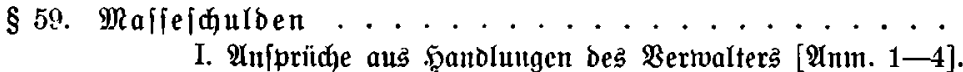

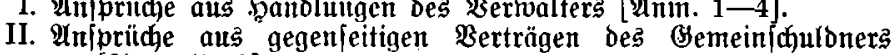
[थีm. 5-9].

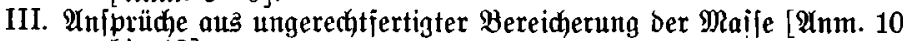
bis 12].

IV. Internationales $\Re$ edyt: fiehe $\$ 57$.

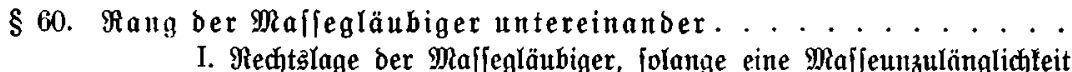

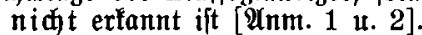

II. Rechtšlage ber Maffegläubiger, nađ̆b em bie Maffeunzulängliđfét erfannt ift [भinm. 3-9].

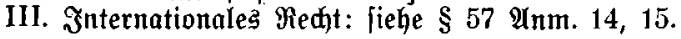




\section{Sonturagläubiger.}

§61. Rangoronung ber Roufursgläubiger (Borrecte) . . . . . . 847-872

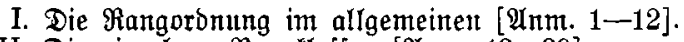

II. Die einzelnen $\Re$ angflaffen [2̈nm. 13-39].

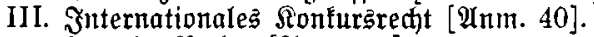
Эrembe :Redte [Unm. 41].

$\S 62$. Rang ber Rebenjorberungen . . . . . . . . . . . . 872-874

I. Die Roften [AHm. 1].

II. Die Bertragêtrafen [9um. 2 u. 3].

III. Die Binfen [थ̈m. 4].

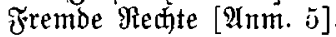

$\S 63$. Unanmeldbare Forderungen . . . . . . . . . . . . . 874-880

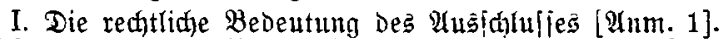

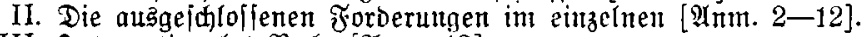

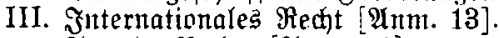

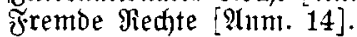

$\S 64$. Abjonberunģbereftigte fionfurggläubiger . . . . . . . . 880-888

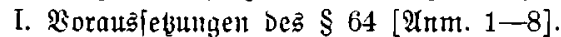

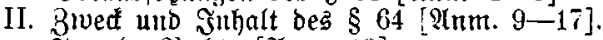
Frembe Piechte [Anmı. 18].

$\S 65$. Betagte Soukuraforderungen

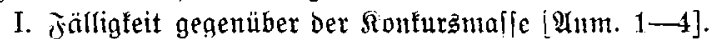

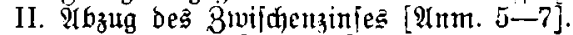

III. Tontofurrent [थ̈m. 8].

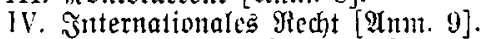
Frembe Redite [भnm. 10].

\$66. Ronfuraforberungen unter aujlöender Bebingung. . . . . . . 893-894

I. Grumbiäbliche 8 ulaffung [9um. 1].

II. Eintritt Der \&eningung $[\mathfrak{A} n$ m. 2].

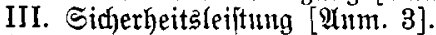

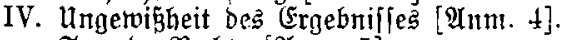

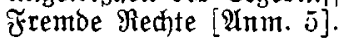

$\S 67$. Ronfursforberungen unter auffolebender Bedingung . . . . . 895-900

I. Begriff ber auffuiebenden Bebingtheit [äm. 1].

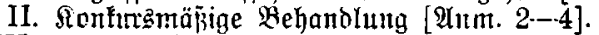

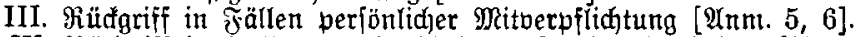

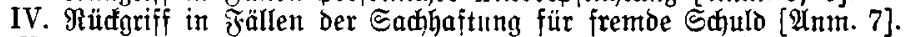

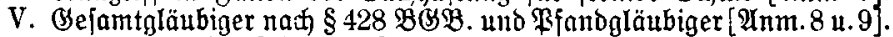

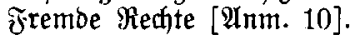

§68. Sonfurşgläubigerređtt fraft Belanthaftung . . . . . . . . . . 900-906

I. Brutbgedante des $\S 68$ [थ̈m. 1].

II. Borausłeţungen mto Inthalt im einzelnen [A̛nm. 2-7].

III. Buvielempfang [âm. 8].

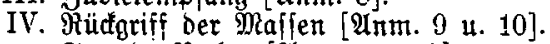

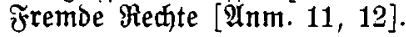

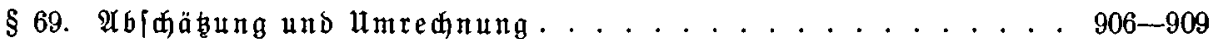

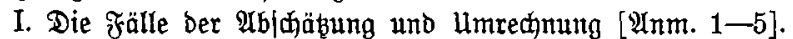

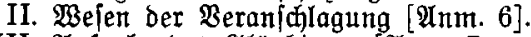

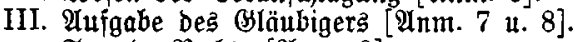

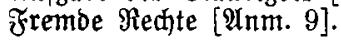

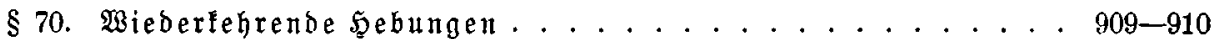




\title{
3weiter Band.
}

\author{
3weites $\mathfrak{B}$ ud. \\ finkntwerfahxen.
}

Eriter Titel.

MIIgemeine Bejtimmungel. Eeit:

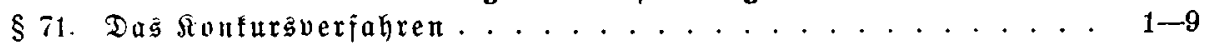

I. Begriff und Bejen des Sonfurşberfahrens [91nul. 1-7].

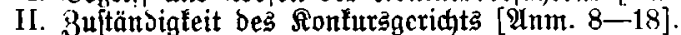

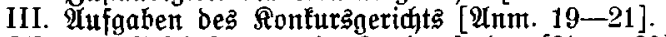

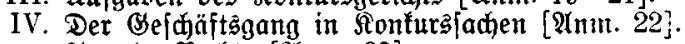
Frembe Fedfte [थ̈nm. 23].

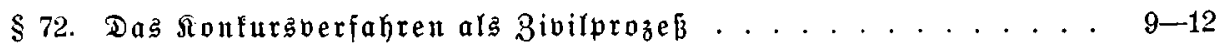

I. Bejonderheiten [थ̈nm. 1-6].

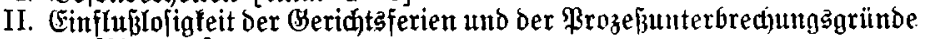
[:̈nm. 7].

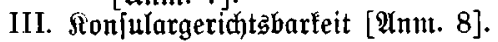

$\S 73$. 3uftellungen . . . . . . . . . . . . . . . . . . . 12-17

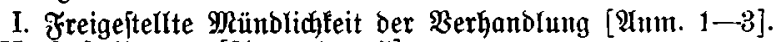

II. Buitellungen [ânm. 4 a. 5].

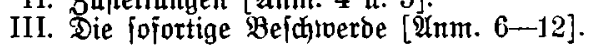

§ 74. Entideibung bes Befdwerbegeridts . . . . . . . . . . . . 17-19

$\S 75$. Ermittelungen von $\mathfrak{A} m \mathfrak{m} \mathfrak{s}$ wegen . . . . . . . . . . . . . 19-21

I. Sffizialprinzip [Q̛⿱nm. 1].

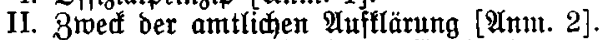

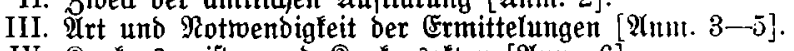

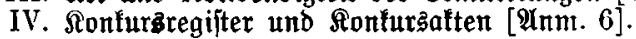

$\S 76$. Dffentlide $\mathfrak{B e f a n n t m a d u n g e n . \ldots . . . . . . . . . . . ~ 2 2 - 2 3 ~}$

I. Dffentlidue Befanntmaduungen [A̛m. 1].

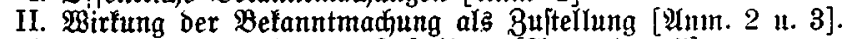

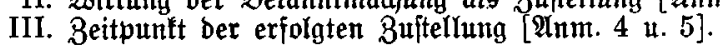

§ 77. $3 u$ itellungen und Mitteilungen . . . . . . . . . . 23-24

I. Cinzelz̆uftellungen [भ̆nm. 1].

II. Mitteilungen [A्Am. 2].

§ 78. Der Ronfurivertwalter . . . . . . . . . . . . . . . 24-31

I. Das $\mathfrak{A m t}$ deș ßerwalters [ânm. 1-10].

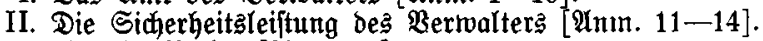

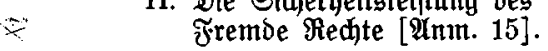

$\S 79$. Mehrere Beriwalter . . . . . . . . . . . . . . . 31-32

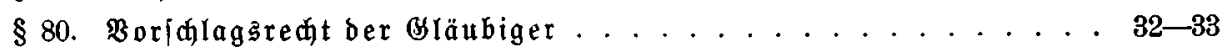

§ 81. Beftellung bes Berivaltera . . . . . . . . . . . . . . 33-34

I. $\mathfrak{A}$ mtß̈antritt $[\mathfrak{A} \mathfrak{n m} .1]$.

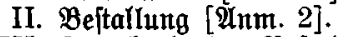

III. 3urüfgabe ber $\mathfrak{B e f t a l l u n g}$ [9̈nm. 3 u. 4'. 
\$ 82. Beriönlidue Berantwortlidfeit dę Bertwalter

I. Begriff Der Berantwortlichfeeit [भ̂n. 1].

II. Berantroortlidfleit gegenüber allen Beteiligten [ $\mathfrak{A n m}$. 2-j]

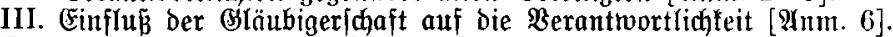

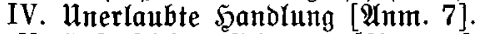

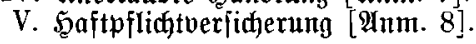

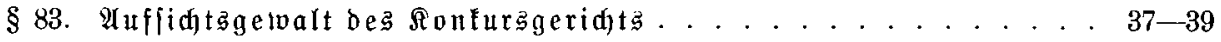

$\S 84$. strafell .................... . $39-40$

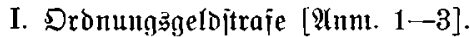

II. Entlafiung [ånm. 4-6].

§85. Aluslagen uno 5̧onorar des Bertualters.

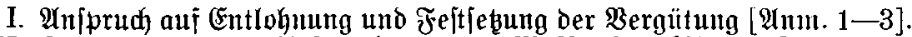

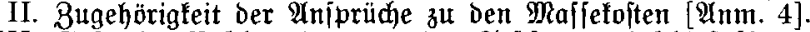

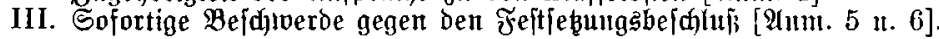
Frembe Tiedte [Anm. 7].

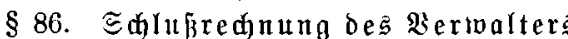

I. Redhenidjaftzablegung [Q̈nn. 1].

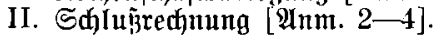

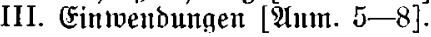

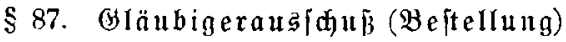
$48-51$

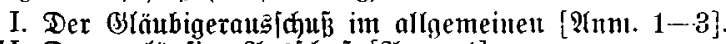

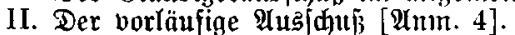

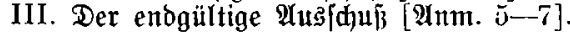
Frembe Rechte [थ́tnm. 8].

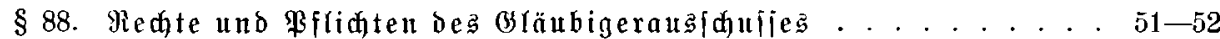

I. Brandiab [înu. 1].

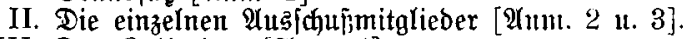

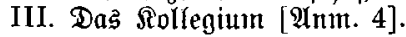

$\S 89.5 \mathfrak{g} a f t u n g$ der Mitglieber . . . . . . . . . . . . . . 53-54

$\S 90$. Rollegialbefdlïfle . . . . . . . . . . . . . . . 54-55

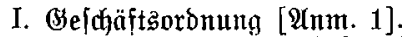

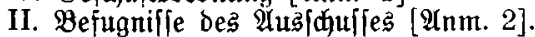

III. Ábjtimmung int eigener Eache [Ömm. 3].

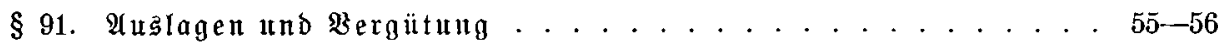

§92. Ende ber Migliedfdaft . . . . . . . . . . . . 56-57

§93. Berufung ber bräubigerberjammluug . . . . . . . . . . $57-60$

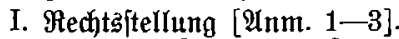

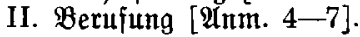
Frembe Redjte [:̈nm. 8].

§94. Reitung und Befdhlǘfaffung

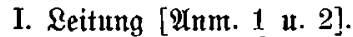

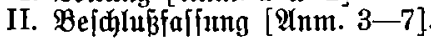
Frembe Reçte [A̛mm. 8].

§ 95. Stimmreqt ber Gläubigerberfammlung . . . . . . . . . 62-64

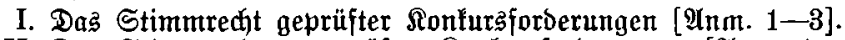

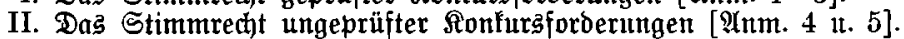

III. $\mathfrak{A b}$ timmung in eigener Sadhe [A̛m. 6].

§96. ङtimmredt in"Der Gläubigerveriammlung

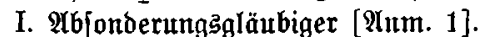

II. Bebingte Forberungen [भinm. 2].

III. Befondere Borredte [थ̈m. 3 u. 4]

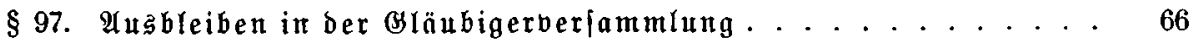

§98. Tagezoronung der Ğläubigerberfammlıng. . . . . . . . . 66 
§99. Beto des Beridts . . . . . . . . . . . . . . 67-68

I. Boraus]ebungen [A̛m. 1-4].

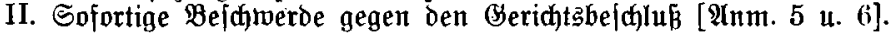

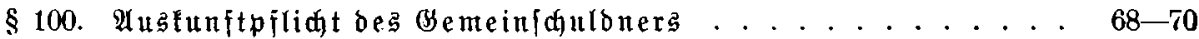

I. Regelfall $[\mathfrak{A n m} .1-3]$.

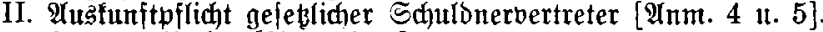
Frembe Redite [âm. 6, 7].

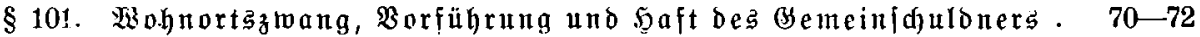

I. $\mathfrak{B}$ ofnortszzong [भinn, 1$]$.

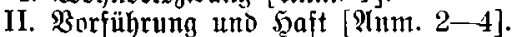

III. Blwang gegen geleblidje Ed)uldnervertreter [?Inm. 5]. Fremoe Fiedte [?tim. 6].

\section{3weiter Titer.}

\section{Erijinungäveriahren.}

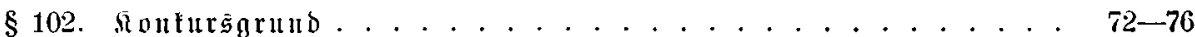

I. Die Stonfuržnorausfebungen im allgemeiten [Amm. 1].

II. Der Sonftrşgrunio im befouderen [भinm. 2-5]. Frembe Redite $[\mathfrak{A}$ um. 6, 7].

$\S 103$. Ronkurzantrag

I. Cröffnung nur auf $\mathfrak{A}$ utrag [थ̈nm. 1 u. 2].

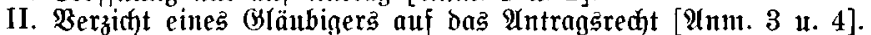

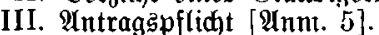

IV. Seilung eines $\mathfrak{A}$ ntragsmangels [Anm. 6].

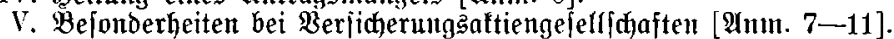
Frembe Redite [Arm. 12].

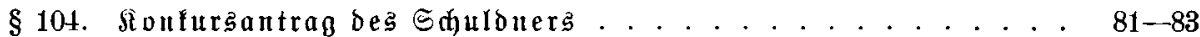

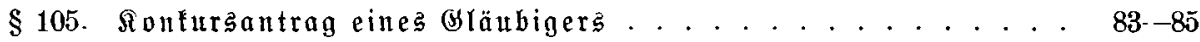

§ 106. Eilltweilige $\mathfrak{A}$ noronungen . . . . . . . . . . . . . . . . . . . 85-90

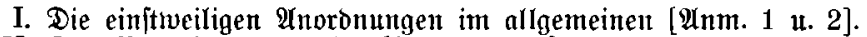

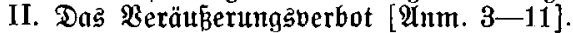

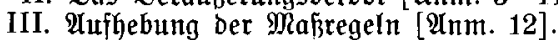

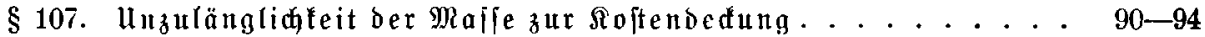

I. Mafiemangel als Ronfurshintbernis [Ân. 1].

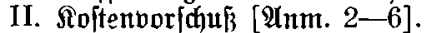

III. Befonderheit [\{̛nm. 7].

IV. Earivarze Rifte [Ânm. 8].

V. Statiftif $[\mathfrak{A} \mathfrak{n m} .9]$.

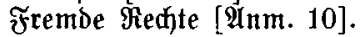

§ 108. 3eitpunft ber $\mathfrak{A}$ onfurzeröffnung . . . . . . . . . . . . 95-98

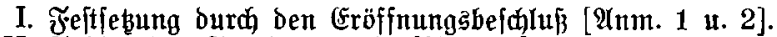

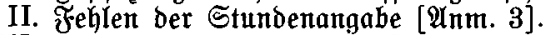

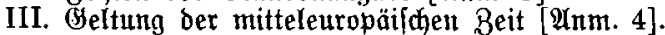

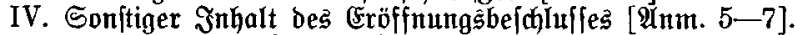

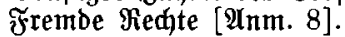

§109. Bejdmerbe gegen (Eröffnung . . . . . . . . . . . . 98-102

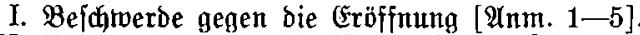

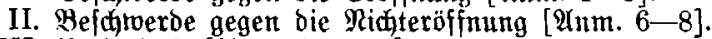

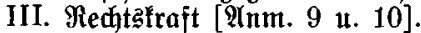
Fremtoe Reatse [Äm. 11].

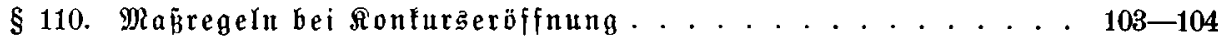

§ 111. Befanntmadung ber fionfuraeröffnung. . . . . . . . . 104-105

$\S 112$. Mitteilung ber $\mathfrak{x}$ onfuräeröffnung an 8 ehörben . . . . . . 105-107 


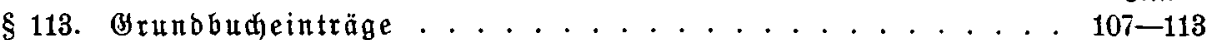

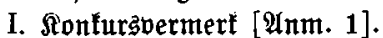

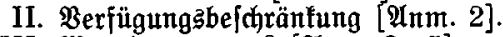

III. $\mathfrak{B O}_{0}$ einzutragen? [ $\left.\mathfrak{A} \mathfrak{n m} .3-5\right]$.

IV. Sofortiger Eintrag [̊̈nm. 6].

V. Gruntobudiperre [Annt. 7-10].

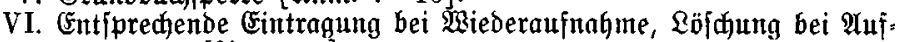
hebung $[\mathfrak{A n m} .11]$.

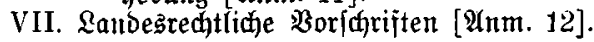

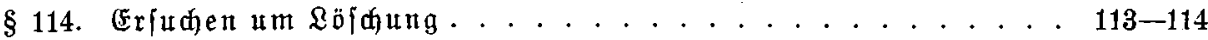

$\S 115$. Gebührenfreie Einträge . . . . . . . . . . . . 114-115

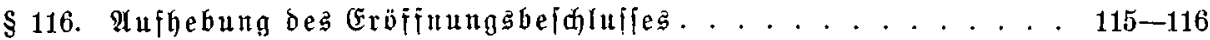

Dritter Titel.

Teilungämaife.

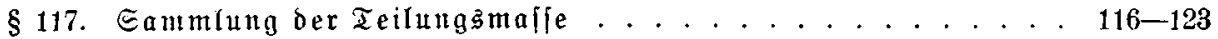

I. Begriff [भน⿰m. 1].

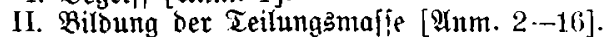

III. Berivertung [भitm. 17-21]. Frembe Fiectite [A1m. 22].

§ 118. Dfjener Arreft . . . . . . . . . . . . . . . 123-125

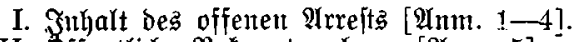

II. Sffentlidise $\mathfrak{B e f a m t m a d ) u n g ~}[\mathfrak{A}$ mm. 5].

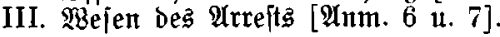

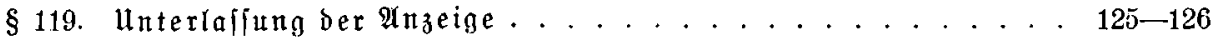

$\S 120$. Borzeigungaplidt ber Ablonderungaberedtigten . . . . . 126-127

§ 121. Boitfperre................... 127-130

I. Fnhalt uno Wirfung [थีmm. 1-5].

II. Sofortige Be[diwerie [भim. 6 u. 7].

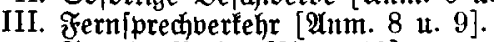

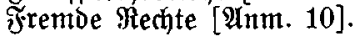

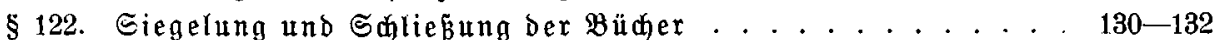

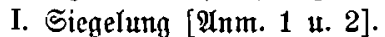

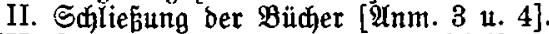

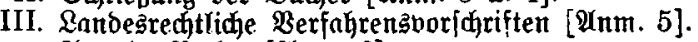

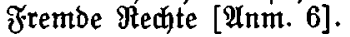

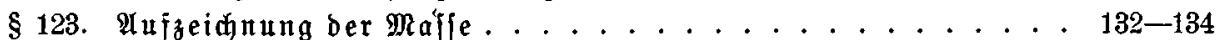

I. Aufzeifnumg [Anm. 1-3].

II. $\mathfrak{A} 1 t \mathfrak{s n a h m e n}[\mathfrak{A} \mathfrak{n m} .4]$.

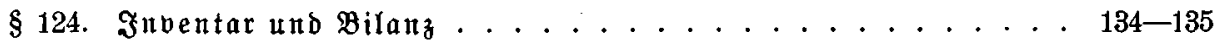

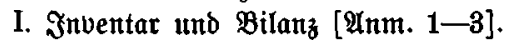

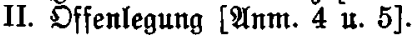

$\S 125$. Dffenbarungzeid . . . . . . . . . . . . . . . 135-139

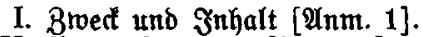

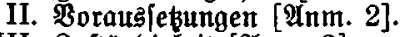

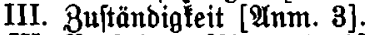

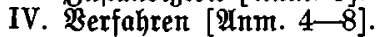

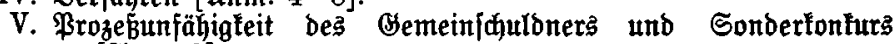
[भinm. 9].

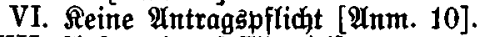

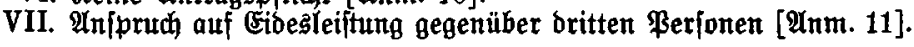

\$126. Bwanggverwertung von Riegenidaften . . . . . . . . . 139-149

I. Die Bebeutung Der $\$ \$ 126,127$ im allgemeinen [भInm. 1-3].

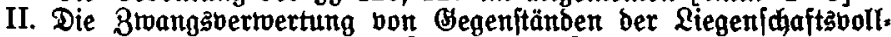
ftretung im befonberen [भInm. 4-16]. 
III. Die ßerwertung von Fahrnis int bejonteren [थ̈nm. 1-15].

$\S 128$. Der Bemeinfduldoner als Borerbe . . . . . . . . . . . 159-163

I. Ratio legis [2Hm. 1].

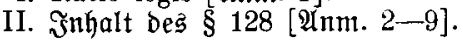

§ 129. Borläufige Má̉nahmen . . . . . . . . . . . . . 163-166

I. Borläufige Regelung oringlicher $\mathfrak{U}$ ngelegenheiten [Änn. 1].

II. Rotoürftiger linterhalt [2̈nm. 2-8].

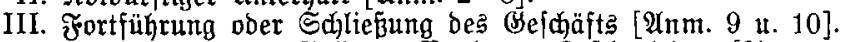

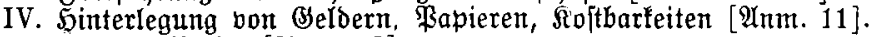
Frembe Recte [भnm. 12].

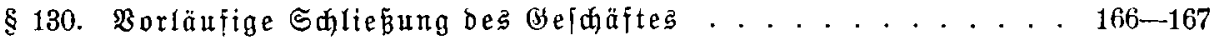

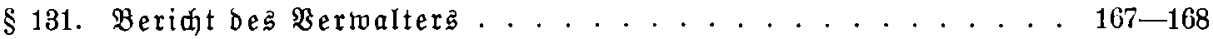

§ 132. Befdlülfe der ßSläubigerverfammlung . . . . . . . . . . . . . 168-169

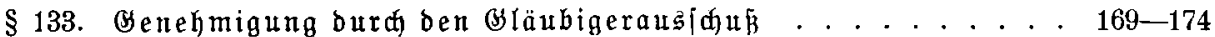

I. Semeinfames ber $\$ \S 133,134$ [थnm. 1-6].

II. Die einzelnen Fälle des $\$ 133$ [थInm. 7-12].

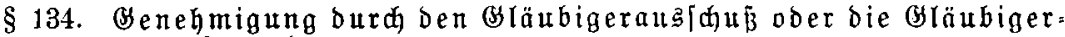

verfammlung. . . . . . . . . . . . . 174-176

III. Die einzelnen Fălle des § 134 [भ̆⿵m. 1-4].

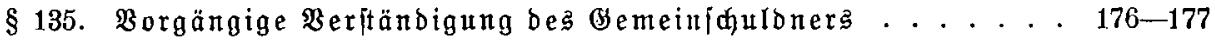

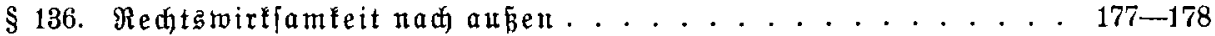

$\$ 137$. Erhebung hinterlegter Berte . . . . . . . . . . . . 178-180

Bierter Titel.

อ̆bulbenmafie.

$\S 138$. Anmelbefrift . . . . . . . . . . . . . . . . 181-183

I. Begriff ber Sduulbenmajfe [Önm. 1 u. 2].

II. $\mathfrak{U} \mathfrak{n m e l}$ efrift $[\mathfrak{a} \mathfrak{n m} .3$ t. 4 ].

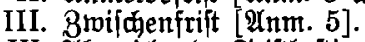

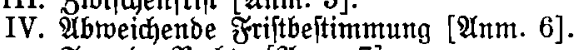

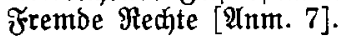

\$ 139. Unmelbungserforberniffe . . . . . . . . . . . . . . 183-192

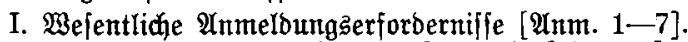

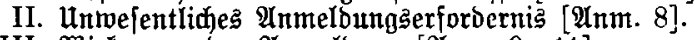

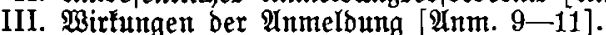

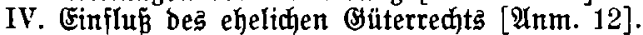

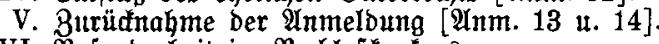

VI. Beionderheit im Rachlab̆tonturs.

$\S 140$. Ronfurztabelle $192-195$

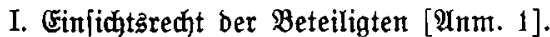

II. Eintraguts in bie Tabelle [भrm. 2-4].

III. Eintidtung Der Tabelle [थ̈nm. 5-10].

$\S 141$. Brüfungstermin . . . . . . . . . . . . . . 196-201

I. 3roef [ $[\mathfrak{A n m} .1]$.

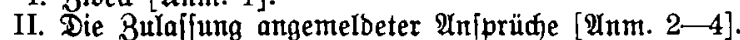

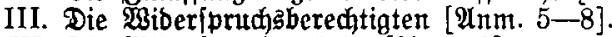

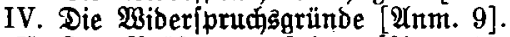

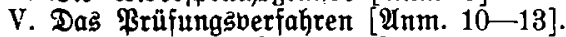

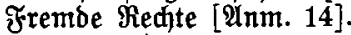

$\S 142$. ßrüfungstermin . . . . . . . . . . . . . . 201-204

I. Der be[ondere $\mathfrak{B r u ̈ f u n g s t e r m i n ~ [ A ̂ n . ~ 1 - 4 ] . ~}$

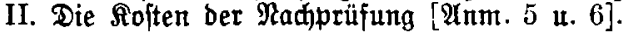

$\S 148$. Uนsbleiben bes Anmelders im $\mathfrak{B r u ̈ f u n g s t e r m i n . ~}$ 


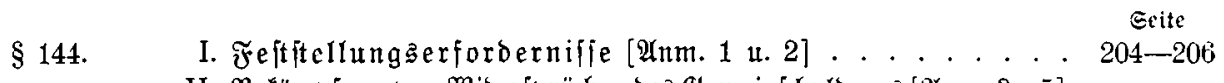

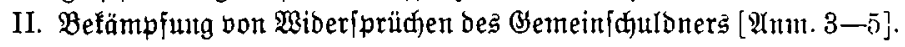

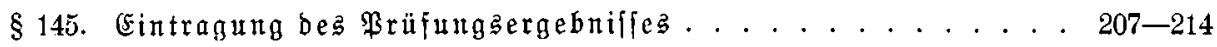

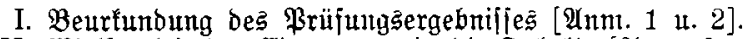

II. Wirffamteit Der (sintragung in bie Tabelle [2ּnm. 3-9].

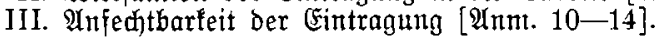

§ 146. Feftitellung beftrittener Forderungen . . . . . . . . . . . . . 215-241

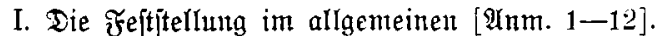

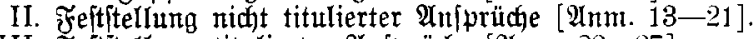

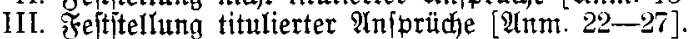

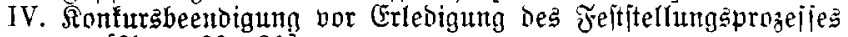
$[\mathfrak{A} 1 \mathrm{~m} .28-30]$.

V. Beridftigung Der Tabelle [?

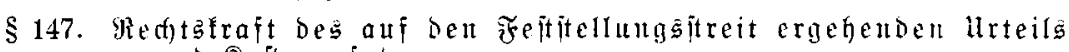

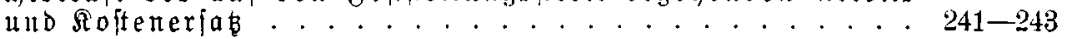

I. Redt)

II. Roltenerfab aus der Mafie [Ünn. 4-8].

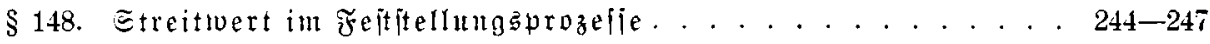

\section{Fün†ter Iitel.}

\section{Berteilung.}

\$149. Beitputt der Berteilungen. . . . . . . . . . . . . . 247-240

I. Die Berteilungen im allgemeinen [:

II. Die Abiallagzberteilung [A्Amm. $\overline{5}-\overline{7}]$. frembe Reditc [Åm. 8].

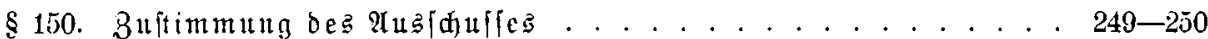

$\S 151$. Antüudigung eiter Berteilung . . . . . . . . . . . . 250-252

I. Berteifungshijte [भ̈nm. 1-7].

II. Die öffentlidge Befarntmađhung [भInm. 8].

III. Beritößze gegen $\S 151$ [थ̈m. 9].

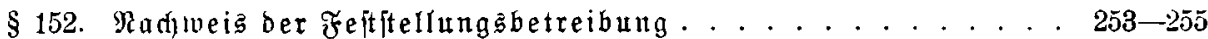

I. Die grundjäbliche Bedeutıng bes $\$ 152$ [भnm. 1 it. 2].

II. Die $\mathfrak{A}$ usłatlusfriit $[\mathscr{U} \mathfrak{m} .3$ u. 4].

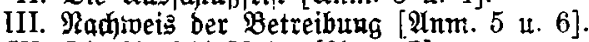

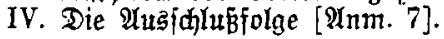

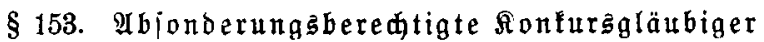

I. Die Regelung im allgemeinen [2rm. 1 u. 2].

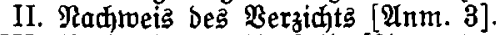

III. Nadjweis bes

IV. Antwenbung auf Borrechtägläubiger [A̛m. 7-10].

§ 154. Bebingte Ronfurşforberungen ............ 258-259

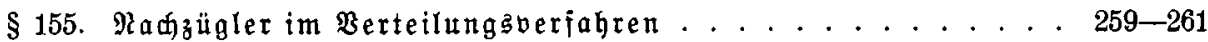

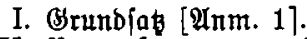

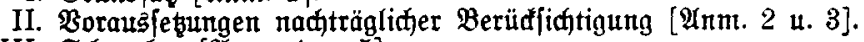

III. Squranten [थ̈nm. 4 น. 5].

IV. Bollzug [भnm. 6].

Frembe Redte [भinm. 7].

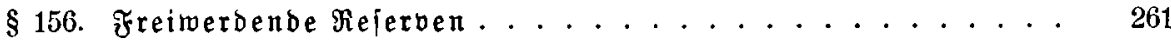

§ 157. Beriđtigung ber Berteilungslifte . . . . . . . . . . 262

$\S 158$. Eintwendungen gegen bie Abidlaģ̧verteilungalifte . . . . 262-266

I. Grundfał $\left[\mathfrak{A}_{\mathfrak{n} m}\right.$. 1].

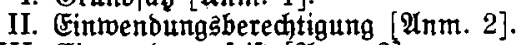

III. Cinwenbung 


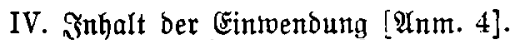

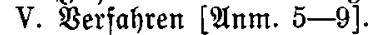

VI. AGlauf ber Einwendungsifritt [Arm. 10].

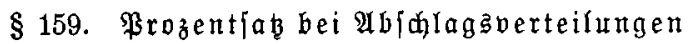

I. Bejtimmung [थ̈rm. 1-3].

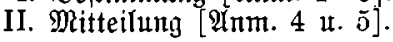

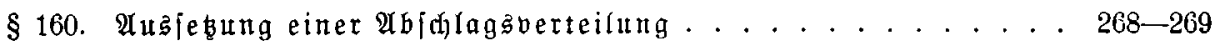

I. Borausleb̨ung [भ̈nm. 1].

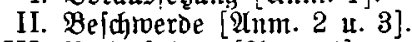

III. Redtiffolgen [Âtm. 4].

IV. Beendigung [înm. 5].

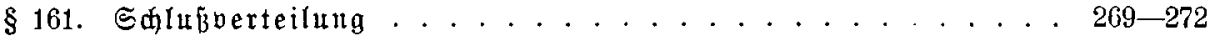

I. Beitpunkt [Änn. 1-5].

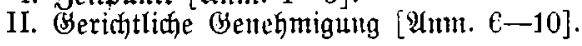

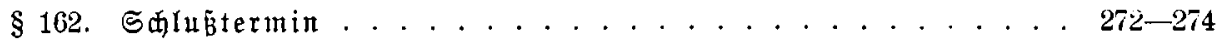

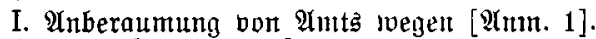

II. 3roef [थ̈m. 2-7].

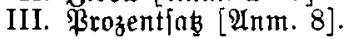

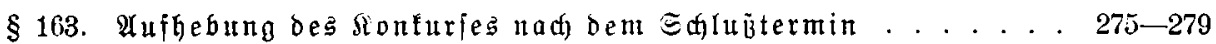

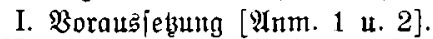

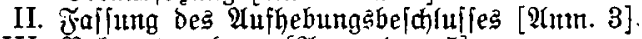

III. Befanntmad)ung [9ּm. 4 it. 5 ].

IV. Redtafolgen [भum. 6-8].

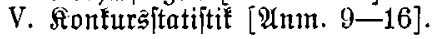

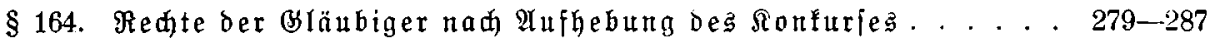

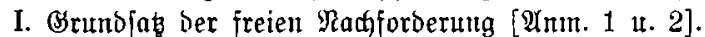

I1. Die Urteilstraft Der Fefttellung gegenüber bem Bemein/duldonter $[\mathfrak{A} \pi \mathrm{m} .3-12]$.

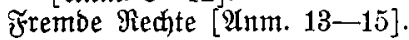

§ 165. Wiebereinfełung gegen Berfäumung oes $\mathfrak{B r u ̈ f u n g s t e r m i n s ~ . ~ . ~ 2 8 7 - 2 9 0 ~}$

I. Ratio legis [Arm. 1].

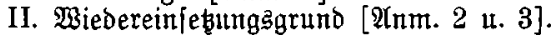

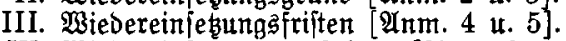

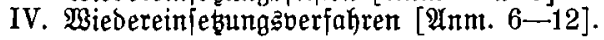

$\S 166 . \Re \mathfrak{a}$ tragguerteilung . . . . . . . . . . . . . . . . . 290-295

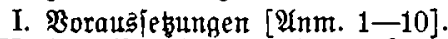

II. Das Berteilungšberfahren [भ̆mm. 11 u. 12].

III. Brenzen Der Befdhlaganadivirfung [ÂAm. 13].

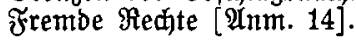

$\S 167$. Berteilungavolrzug . . . . . . . . . . . . . . . 295-296

I. $\mathfrak{A}$ rt und $\mathfrak{B}$ eife [भ̆m. 1].

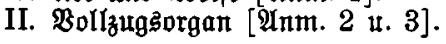

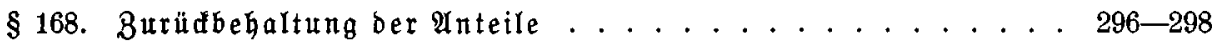

I. Die Burüđtbehaltung im allgemeinen [9ּn. 1 u. 2].

II. Burüđbehaltungęgrünbe [भ̆m. 3-9].

§ 169. Şinterlegung zurüđbehaltener $\mathfrak{A n t e i l e ~ . ~ . ~ . ~ . ~ . ~ . ~ . ~ . ~ . ~ . ~ . ~ . ~ . ~ 2 9 8 - 3 0 0 ~}$

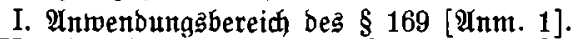

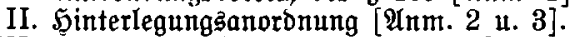

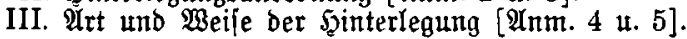

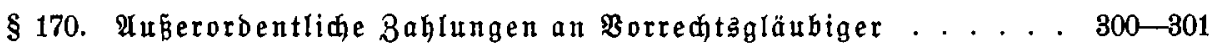

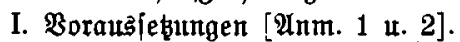

II. Beborreditigte Forberungen [भinm. 3].

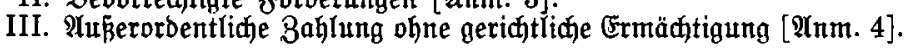

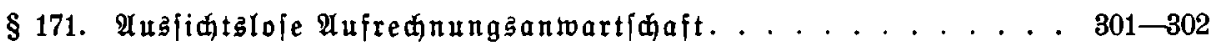




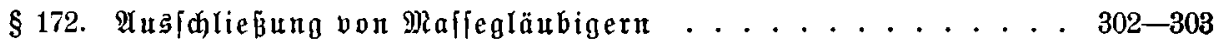

I. Unabhängigleit ber Dedung ber Maffeaniprüche vom Sonturäber:

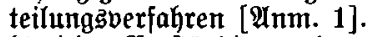

II. Rechtzeitige Berftändigung bes Berwalters [2̈nm. 2-5].

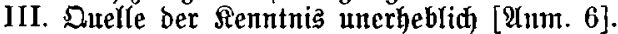

IV. Beltungşbereich des $\S 172$ [थีm. 7].

Sediter Titel.

Bwangabergleid.

§173. Der 31vangšvergleid ................ 304-315

I. Begriff, Bwedf uno Bejen [Anm. 1-17].

II. Statthaftigfeit [भ̈nm. 18].

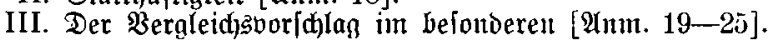

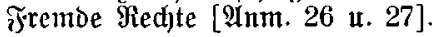

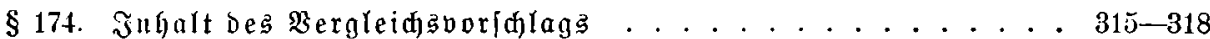

I. Criforberniz Der Bejtimmtheit [91nm. 1].

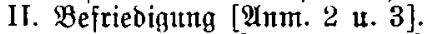

III. Sidieritellung [Ätm. 4 u. 5].

IV. Boridlaģänderungen [Q̈nm. 6]. Frembe Rechte [Üm. 7].

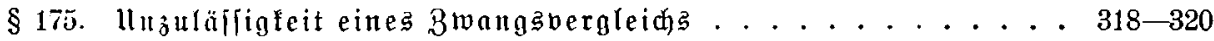

I. Dic Unzuläffigfeit int allgemeinen [भ̆nn. 1-3].

II. Die cinzelnen Gơründe ber hnzuläffigleit [9:mm. 4-8].

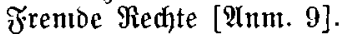

$\S 176$. Freigeftellte 3 urüđiveifung bes Boridlags. . . . . . . . . . 321—322

I. Burüđţveifungsggründe [भ̆nm. 1-3].

II. Ermeifensfrage [ân. 4].

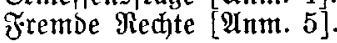

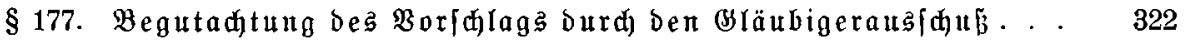

§ 178. Rieberlegung von Borfdlag unb Butadten . . . . . . . 323

$\S 179$. Der Bergleidgtermin . . . . . . . . . . . . . . 323-325

I. Unberaumung bes Termins [भ̈tm. 1].

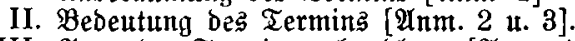

III. Bang ber Terminzerhanolung [थีm. 4].

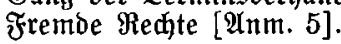

$\S 180$. Termitzoerbindung . . . . . . . . . . . . . . . 326

$\S 181$. Oreichmäßige Befriebigung . . . . . . . . . . . . $326-333$

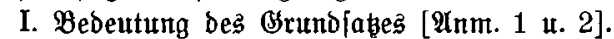

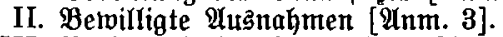

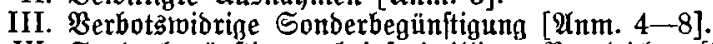

IV. Sonderbegünttigung bei freimilligen Bergleiđen [भ̂nm. 9 u. 10].

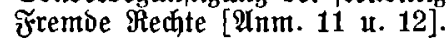

$\S 182$. Die $\mathfrak{A b}$ timmung. . . . . . . . . . . . . . .

I. Ropfmehrheit [ $\mathfrak{A n m} .1-5]$.

II. Summenmehrheit [भ̈nm. 6].

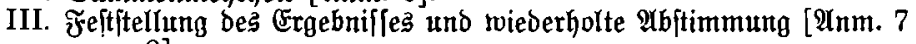
u. 8].

Frembe Redte [̊nm. 9 u. 10].

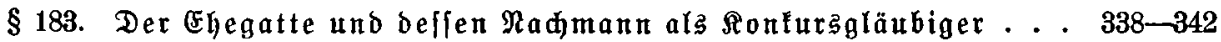

I. Breeff und Beredtigung bes $\S 183$ [भinm. 1 u. 2].

II. Der Jnhalt bes $\$ 183$ [थ̈m. 3-8].

III. Âtwendurt im Sonderfonfurle [ânm. 9]. Frembe Redte [भinm. 10]. 


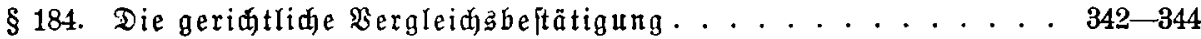

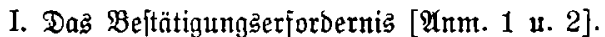

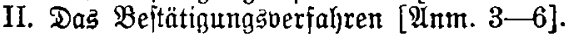
Frembe Rechte [Arm. 7].

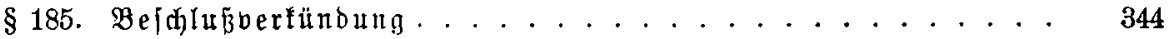

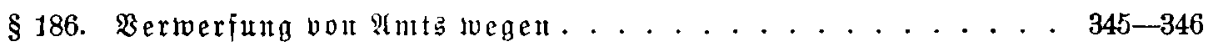

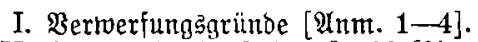

II. Broingende Siraft bes $\$ 186$ [Anm. 5].

$\S 187$. Berwerfung wegen Minderergebniffes . . . . . . . . . . . 346-350

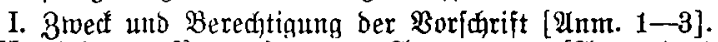

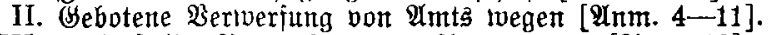

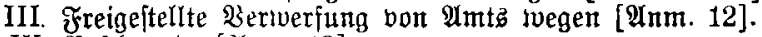

IV. Beigherde [अinm. 13]. Frembe Rechte [9rmm. 14].

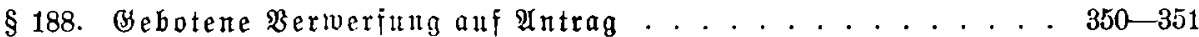

I. Der $\mathfrak{A n t r a g}[\mathfrak{A}$ um. 1-4].

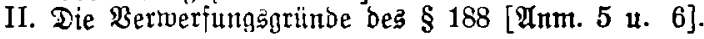

$\S 189$. Befdwerde gegen Beftätigung ober Berwerfung . . . . . . 352-353

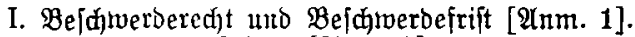

II. Beidmerbeverfigren [:Inm. 2].

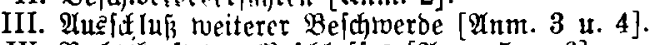

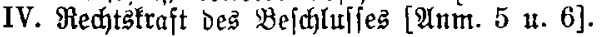

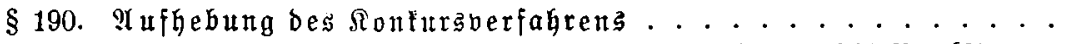

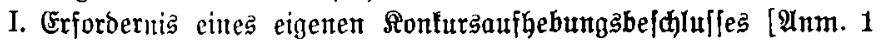
bis 3].

II. Beitpunt ber Sonfursaufhebung [ăm. 4-6].

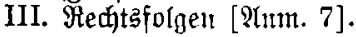

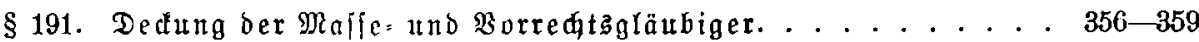

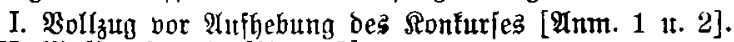

II. Majleanipriiche [Änm. 3].

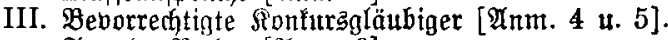
Fremtie Redte [Arm. 6].

$\$ 192$. Wieberḱk̆r ber Berfügungafreiheit . . . . . . . . . . . 359-362

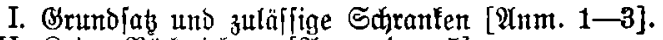

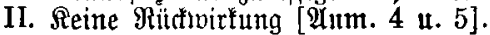
Frembe Redte [थ̈nm. 6].

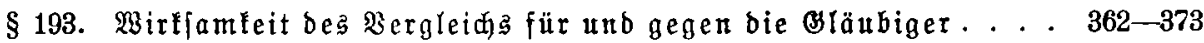

I. Einfluß alf bie nidht bevorredtigten Ronfuräforberungen [Q̈nm. 1 bis 4].

II. Die Reftiduulb als unvollfommente Berbinblidfeit [M্nm. 5 u. 6].

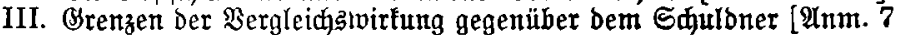
bis 13].

IV. Unberührte 5jaftung Dritter [Ünm. 14-18].

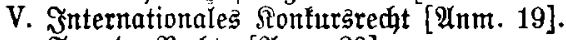

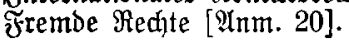

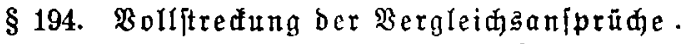

I. Der Bollitrecfungstitel [ânm. 1-3]

II. Bergleidgsgaranten [ $\mathfrak{A m} .4-6]$.

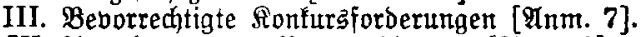

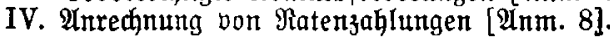

$\S 195$. Riđterfüllung bes 3 wangzvergleiđs. . . . . . . . . . 377-379

I. Die Bergleid]కెd

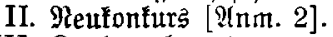

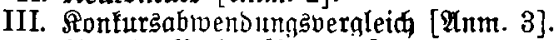
Fremde Red)te [2(tum. 4]. 
$\S$ 196. Anfedtung beş Erlaifes wegen Betrags . . . . . . . . . . . 379-382

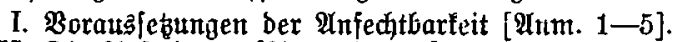

II. Die $\mathfrak{A n f e d t u n g}[\mathfrak{A} \mathfrak{n m} .6 \mathfrak{u}$. 7].

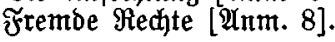

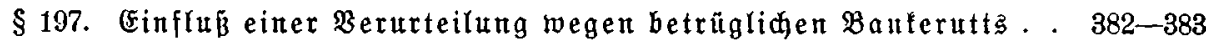

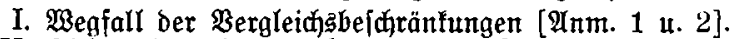

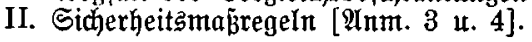

$\S 198$. Wieberaufnahme bes $\mathfrak{R}$ onfurfes . . . . . . . . . . . . . . 383-387

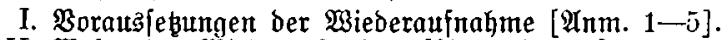

II. $\mathfrak{B e f e n}$ Der $\mathfrak{B i e d e r a u j n a h m e ~ [ \mathfrak { A n m } . 6}$ u. 7].

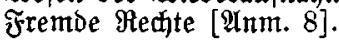

$\S 199 . \quad E r\{a b$ ber $3 \mathfrak{a} \mathfrak{l}$ lungzeinftellung . . . . . . . . . . . . . 387-388

$\$ 200$. Die Brläubiger bez aufgenommenen Berfahrenz. . . . . . . . 388-389

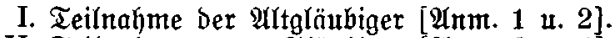

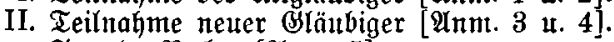
Frembe Rehte [Äm. 5].

$\S 201$. Berfahren nad ber $\mathfrak{B i e d e r a u f n a h m e ~ . ~ . ~ . ~ . ~ . ~ . ~ . ~ . ~ . ~ 3 9 0 - 3 9 1 ~}$

I. Brand $[a \mathfrak{b}[\mathfrak{A} n$ m. 1 u. 2$]$.

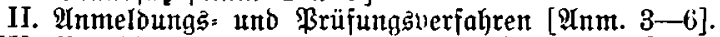

III. Beendigung bes aufgenommenten Berfahrens [Anm. 7].

\section{Siebenter Titel.}

cinftellung beg Beriahreng.

$\S \S 202,203$. Sonfursverzidyt . . . . . . . . . . . . . . . . 391-396

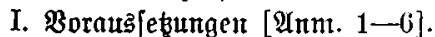

II. Berfahren [9̊nm. 7-11].

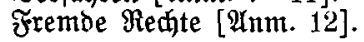

§ 204. Einftellung wegen Mangels der Seftendeđung . . . . . . . . 396-398 $\S \S 205,206$. Bemeinfame Borfariften für beibe Fälle ber Einftellung. 398-401

Imter Titel.

\section{Bejonbere Bepitimmungen.}

$\S \S 207,208$. Sonfurß ber $\mathfrak{A}$ tiengefellfhaft . . . . . . . . . . 401-426

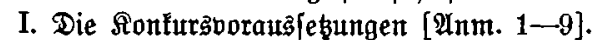

II. Der BSemeinjđulbner [Ünm. 10-13].

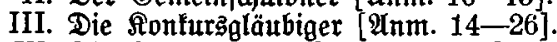

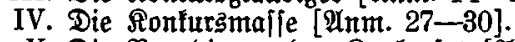

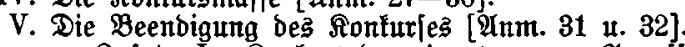

Bufak I. Ronturs ber eingetragenen Benolfenidjaften $[\mathfrak{A} n \mathrm{~m} .33-49]$.

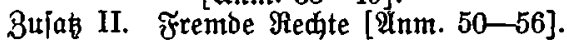

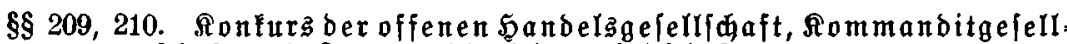

fidaft und Rommanditatiengeferrfaft ....... 426-435

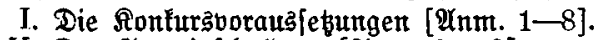

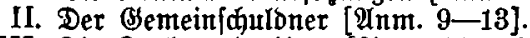

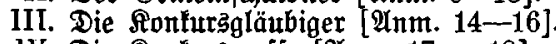

1V. Die Aonfursmalfe [Ânm. 17 u. 18].

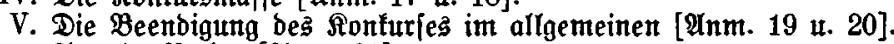

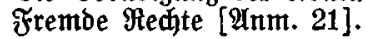

§211. VI. Die Beendigung bes_ßonfuries burd Bwangsvergleid.

\$212. VII. Die Ronfurfe ber perfönlid haftenden Befellíafter

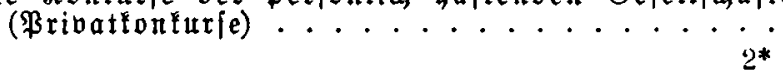


§213. Ronkura von juriftifhen ßerlonen und von Bereinen ohne

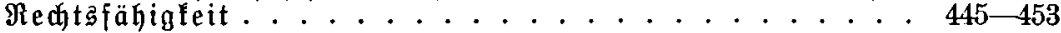

I. Ronfurs jurifitifuer Berfonen [Q̂Anm. 1-13].

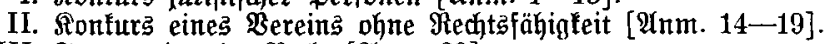

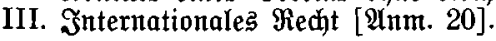

§214. Der Rad,lab̉entur

A. Şm allgemeinen.

I. Begriff uno $\mathfrak{B}$ e[en [Anm. 1-6].

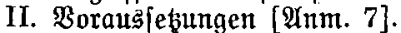

III. Bemeinf dulloner [Ânm. 8-20]

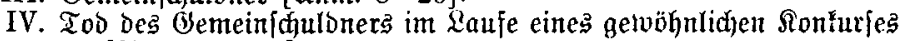
[अ̈m. 21-24].

V. Die Sonfursmalfe [Ânm. 25-31].

B. Die einzelnen Bor[đriften ber $\Re 5$.

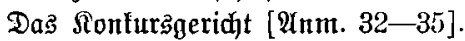

$\S 215$. Der $\mathfrak{i}$ onfurḡgrund . . . . . . . . . . . . . . . 473-474

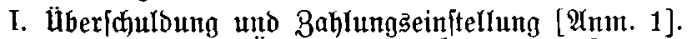

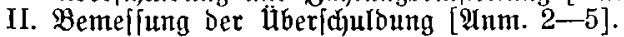

$\S 216$. Sonfurzmöglidłeit . . . . . . . . .

I. 2ei unbe[dränfter Erbenhaftung [9rnm. 1-3].

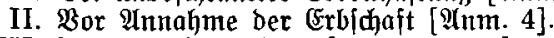

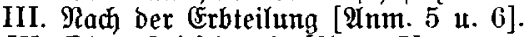

IV. Dhne Beitjdranfe [Q̈nm. 7].

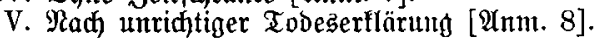

$\S \S 217-220$. §onfurgantrag . . . . . . . . . . . 478-489

I. Antragsberedtigung [9̛nm. 1-25].

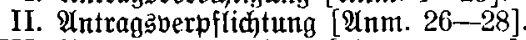

III. Eröffnutgsverfahren [थีm. 29-35].

$\S 221$. Befonderheiten . . . . . . . . . . . . . . 489-494

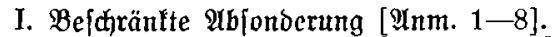

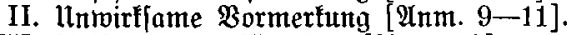

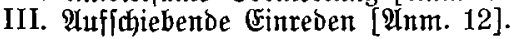

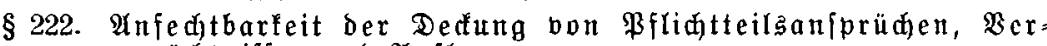

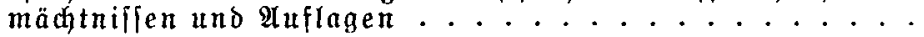

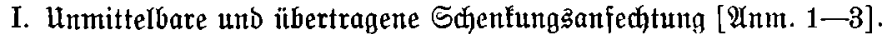

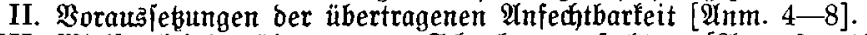

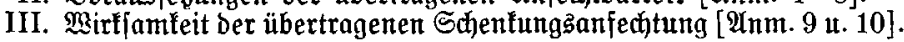

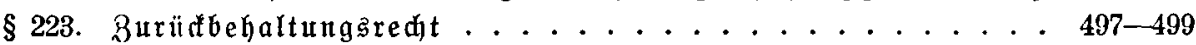

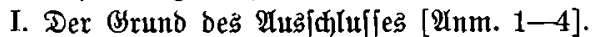

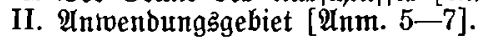

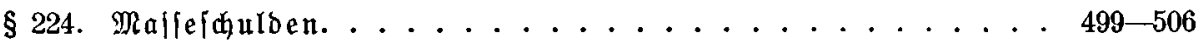

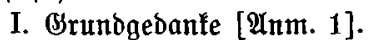

II. Die Malfefdulben des $\$ 224$ im einzelnen [थ̈m. 2-16].

III. Stellung ber $\mathfrak{M a f f e g l a ̈ u b i g e r ~ b e s ~} \$ 224$ [भimm. 17].

IV. Der Erbe als Mallegläubiger [Önm. 18].

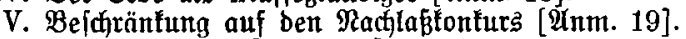
Frembe Rehte [भinm. 20].

$\S 22$ o. $\mathfrak{A} \mathfrak{n}\lceil$ prü

I. Torderungen bes Grben gegen Den Erblaffer [भInm. 1-3].

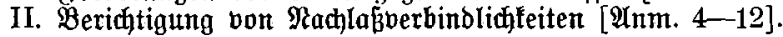

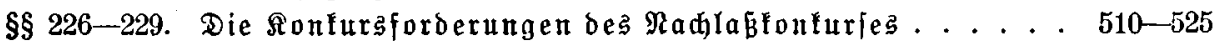

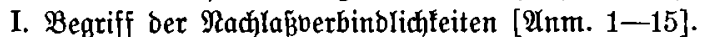

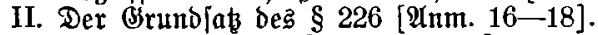

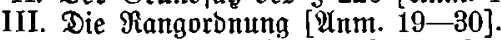

IV. Die minberberechtigten Ronfursforberungen im befonderen [थ̈m. $31-45]$ 


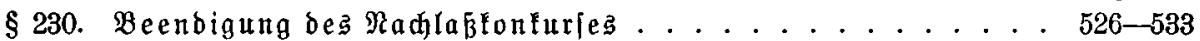

I. Эm allgemeinen [भinn. 1-9].

II. Der Zivangabergleid im bejonberen [A̛nm. 10-19].

$\S 231$. Borerbe und $\mathfrak{R a d}$ erbe . . . . . . . . . . . . . . . . .

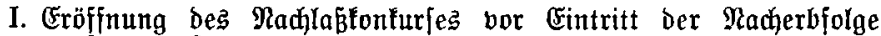
[थีm. 1].

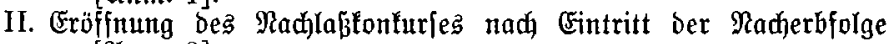
[भinm. 2].

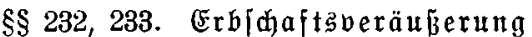
$531-539$

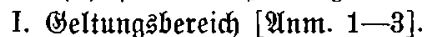

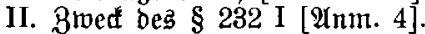

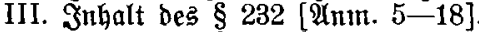

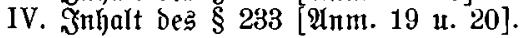

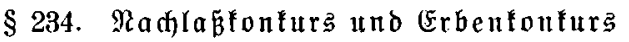

I. Erbenfonfuraz [ânm. 1, 2].

II. Sonturs über Dns Bejamtwermögent bes (5rben $[\mathfrak{A n m} .3-5]$.

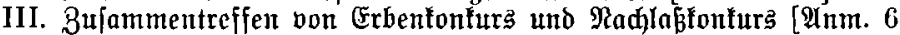
bis 11].

IV. Die Ehefrau als Erbin [थ̈nm. 12-14].

$\S 235$. Mehrheit von 5 rben $\ldots \ldots \ldots \ldots \ldots$

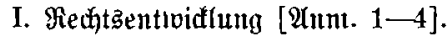

II. Einl)eităfonfurs [2(nm. 5 it. 6].

$\S 236$. Der Befamtgut引lontura . . . . . . . . . . . . 546-553

I. Begriff und Bedeutung [भ̈m. 1-8].

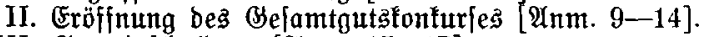

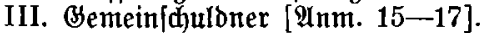

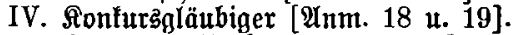

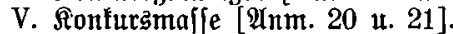

VI. กonfur

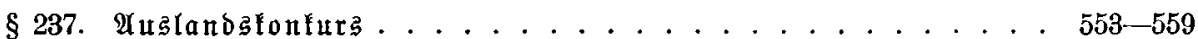

I. Arlgemeine $[\mathfrak{Q}$ nmm. 1 it. 2].

II. $\S 237$ im befonderen [भกm. 3-7].

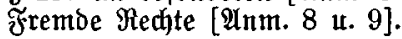

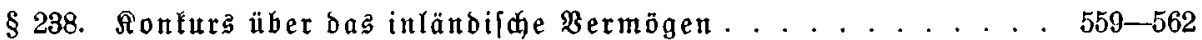

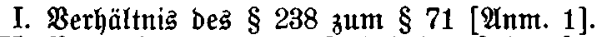

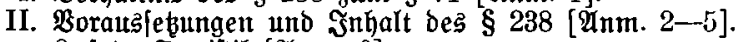

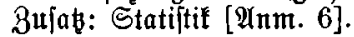

\section{Drittes $\mathfrak{B} \mathfrak{u} \mathfrak{d}$.}

\section{Grafbelfimmungen.}

Borbemerfung . . . . . . . . . . . . . . . . 565-574

$\S 239$. Betrügliđer $\mathfrak{B a n f e r u t t . ~ . ~ . ~ . ~ . ~ . ~ . ~ . ~ . ~ . ~ . ~ . ~ 5 7 5 - 5 9 1 ~}$

I. Tä̀ter $[\mathfrak{A} \mathfrak{n}$ m. 1-10].

II. Bahlutgaeinftellung und Ronfurseröffnung [Ưm. 11-19].

III. Die Banferutthanthungen [थ̈nm. 20-39].

IV. Bollendung, Ber[u孔, Teilnahme, Bufammentreffen von Banterutt: handlungen, Strafe [भ̈nm. 40-52].

§240. Einfacher Banferutt ...

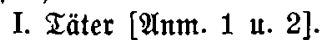

II. Bahlungseinftellung und Sonfurßeröffnung [थ̛m. 3].

III. Die Banferutthandlungen [Üm. 4-41].

IV. Bollendung, Berfuc, Teilnahme, Bu[ammentreffen von Banterutt. hattolungen, Strafe [भinm. 42-49]. 
§ 241. GIäubigerbegün「tigung . . . . . . . . . . . . . 606-613

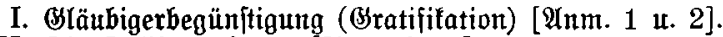

II. Die Deliftzperionen [Äm. 3-9].

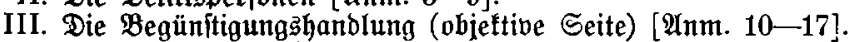

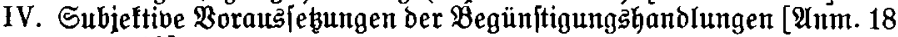
น. 19].

V. Bollendutg, Ber[udh, Teilnahme, Bu[ammentreffen von Begün[ti: gutgahandungen, Strafe [A̛m. 20-27].

$\S 242$. ganleruttunterftüßung . . . . . . . . . . . . 613-616

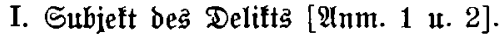

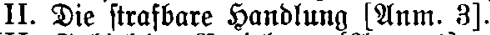

III. Subjeftive $\mathfrak{B}$ eziehung [Änm. 4].

IV. Bollenoung, Berjuh, Teilnahme, 3ujammentreffen, Strafe [शిmm. 5-12].

$\S 243$. Stimmtauf

I. Die Deliftsper[onen [Anm. 1-3].

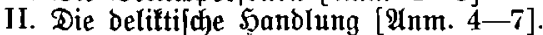

III. Bollendung, Berfud,, Teilnahme, Straje [Âm. 8-11].

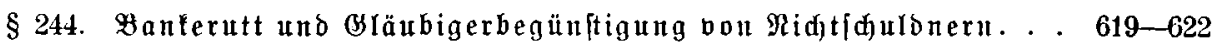

I. Subjefte [ $\left.\mathfrak{A n m}_{\mathbf{n m}} 1-7\right]$.

II. Bahlumgseinftellung und Sonfurseröifnung [भ̆ım. 8].

III. Sit Strafe bedrohte Sandlungen [Äm. 9-12].

\section{II.

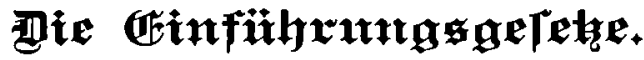

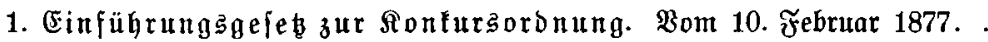

2. Einführungagefeb zu bem Belebe, betreffend änderangen ber R̃onfurzoronung. Bom 17. Mai $1898 \ldots \ldots$

\section{III.}

\section{Anthang.}

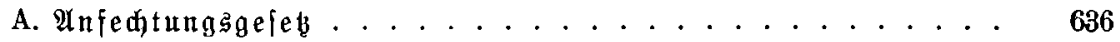

B. Boftengeleze.................... 639

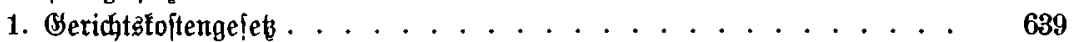

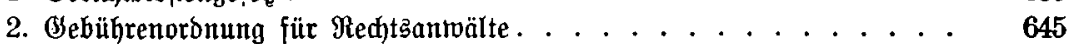

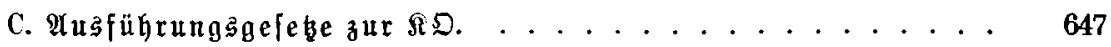

D. Befđäftzorbnungen . . . . . . . . . . . . . 660

1. ßreufien . . . . . . . . . . . . . . . 660

2. Banern ....................... 671

Ulphabetifhes Regifter ................. 683 